\title{
An investigation of the behavior of strain-hardening cement-based composites (SHCC) obtained in a split Hopkinson tension bar and the influence of structural inertia
}

\author{
Ali A. Heravi ${ }^{1 *}$, Joško Ožbolt ${ }^{2}$, Viktor Mechtcherine ${ }^{1}$ \\ ${ }^{1}$ Technische Universität Dresden, Institute of Construction Materials, 01062, Dresden, \\ Germany \\ ${ }^{2}$ Universität Stuttgart, Institute of Construction Materials, 70569, Stuttgart, Germany \\ *Corresponding author
}

\begin{abstract}
The performance of a normal-strength SHCC under impact loading was studied using the results obtained from a split Hopkinson tension bar (SHTB). The focus of the investigation is to explain the mechanisms behind the peculiar rate-dependent behavior of SHCC under tensile loading. With the help of frames obtained by high-speed cameras and the subsequent Digital Image Correlation (DIC) analysis, the stress-strain relation of the SHCC obtained in SHTB was analyzed. The investigation of the composite's behavior was supported by constituent-level experiments on the non-reinforced matrix of the SHCC and on the fiber-matrix bond.

In the case of the constituent matrix, the well-known apparent increase in the tensile strength of the cement-based matrix and its influence on the behavior of SHCC was studied. For this purpose, experiments on the SHCC specimens with different geometries were performed in the SHTB. The results obtained from these experiments and those obtained by DIC show that commonly used analytical models, in which the specimen is assumed elastic, cannot capture the effects of structural inertia on the results. Thus, an alternative novel method based on the results of DIC has been used to explain and quantify the contribution of structural inertia. The rate-dependent behavior of the fiber-matrix bond was studied by performing high-speed single fiber pullout tests in a miniaturized split Hopkinson tension bar. This novel experimental technique enabled explanation of the rate-dependent bridging action of the fibers in SHCC. Based on the results, the enhanced behavior of SHCC under impact loading is explained.
\end{abstract}

Keywords: fiber reinforcement; SHCC; ECC; impact testing; split Hopkinson tension bar; structural inertia 


\section{Introduction}

Strain hardening cement-based composites (SHCC) made of short, randomly distributed fibers show promising behavior with high energy absorption under tensile loading [1], [2]. They can be seen as material solutions for strengthening existing structures against impact loading [3]. Caused by multiple cracking, the quasi-ductile behavior of these composites makes them suitable for the reinforcing layers on the impact-far side of the structures, where tensile stresses are generated and high energy absorption is needed to prevent scabbing of the concrete [4]. Few studies have been published to demonstrate the promising behavior of various types of SHCC under impact tensile loading [5]-[8]. However, the rate-dependent behavior of SHCC and the influence of its constituent elements, i.e. matrix and fiber-matrix bond, on the behavior of the composite still need further investigations.

The split Hopkinson tension bar (SHTB) is a reliable testing method in investigating the behavior of materials within the impact range of strain rate, and has also been used to characterize SHCC [5], [6]. The stress-strain relation obtained at high strain rates by SHTB is affected by the rate-sensitivity of the constituent elements of these composites and their interactions. While first-crack stress is attributed mainly to the tensile strength of the matrix, the multiple cracking behavior is controlled by the cracking stress of the matrix as well as the bridging behavior of the fibers [5], [9]. Thus, a thorough investigation of the rate-sensitivity of the cement-based matrix and its bond to the fiber is essential to explain the behavior of SHCC at high strain rates.

In order to explain the influence of the rate-dependent cement-based matrix on the tensile behavior of SHCC, the mechanism behind the pronounced increase in tensile strength of the matrix at high strain rates should be first explained. The available research points out that the tensile strength of cement-based matrices at high strain rates is not entirely related to material rate-sensitivity. For strain rates higher than $1 \mathrm{~s}^{-1}$, the contribution of the inertia-related mechanisms is considered to be predominant [10]. Nonetheless, conclusive experimental research on the issue is still not available. Several publications have suggested that, unlike compressive strength [11], the lateral-stress-dependent behavior of cement-based material is not the reason behind the considerable increase in the tensile strength at high strain rates [12], [13]. Alternatively, damage growth slower than the loading rate in the specimen is introduced as the main mechanism behind the distinctly higher tensile strength at strain rates greater than $1 \mathrm{~s}^{-1}$. Inertial forces at the crack tip are responsible for the delayed damage in the specimen [14], [15]. Furthermore, structural-level inertia caused by the damage and softening of the material 
in the specimen was introduced in [16]-[18] as a contributing effect to the apparent increase in the tensile strength of cement-based matrices at strain rates higher than $1 \mathrm{~s}^{-1}$. While the limited damage growth rate in the material and the resulting increase in the material strength can be considered using the rate-sensitive macroscopic material constitutive law, structural level inertia needs to be extracted from the experimental results. Otherwise, misinterpretation of structural-inertia as rate-dependent material behavior can lead to an overestimation of the material behavior at high strain rates. So far, the experimental investigations on the influence of structural inertia in split Hopkinson bar experiments are limited to the compressive behavior of cement-based materials. An experimental study on the influence of structural inertia is essential for interpreting the results obtained in the split Hopkinson tension bar.

In this paper the behavior of SHCC under impact tensile load and its fracture mechanism are explained with the help of high-speed stereo imaging at a frame rate of $160000 \mathrm{fps}$ and of the subsequent 3D DIC analysis. Moreover, the contribution of structural inertia to the apparent increase in tensile strength of the cement-based matrix at high strain rates was investigated. For this purpose specimens made of the same normal-strength SHCC, but different geometries, were tested at a high strain rate. Cylindrical specimens with a length of $40 \mathrm{~mm}$ and a diameter of $22 \mathrm{~mm}$ were used as reference. Specimens with a shorter length of $20 \mathrm{~mm}$ but of the same diameter as the reference and annular specimens with the same length and outer diameter as the reference were used to observe the influence of the structural-level inertia in the results. Finally, a novel approach based on the results obtained by Digital Image Correlation (DIC) is proposed and used to find the contribution of structural inertia. Tests at the high strain rate were performed in a gravity-driven split Hopkinson tension bar, which has been presented in previous research of the authors [6], [19], [20].

Furthermore, this work provides an investigation of the rate-dependency of the fiber-matrix bond. Single fiber pullout tests were performed in a miniaturized split Hopkinson tension bar [19]. The pullout displacement rate provided by the setup is comparable to the crack-opening speed of the SHCC specimens tested in the SHTB. The results of these experiments are used to explain the rate-dependent behavior of SHCC observed in the multiple cracking stage.

\section{Experimental program at the composite-scale}

\subsection{Material under investigation and specimen preparation}

The SHCC investigated was made of a normal-strength cement-based matrix and highperformance polymer fibers. The matrix contained a high volume of fly ash and had a water- 
to-binder ratio of 0.3 . Short, randomly distributed high-density polyethylene (HDPE) fibers with a length of $6 \mathrm{~mm}$ were used in a volume ratio of 2\%; see Table 1. The physical and mechanical properties of the fibers are given in Table 2, according to the information received from the producer.

Table 1: Composition of the SHCC under investigation.

\begin{tabular}{|l|l|}
\hline SHCC & {$\left[\mathrm{kg} / \mathrm{m}^{3}\right]$} \\
\hline Cement CEM I 42.5R-HS & 505 \\
\hline Fly ash Steament H4 & 621 \\
\hline Quartz sand 0.06 - 0.2 mm & 536 \\
\hline Viscosity modifying agent & 4.8 \\
\hline Water & 338 \\
\hline Superplasticizer ACE 30 & 10 \\
\hline UHMWPE fiber Dyneema SK62 $(6 \mathrm{~mm})$ & 20 \\
\hline
\end{tabular}

Table 2: Physical and mechanical properties of HDPE fibers

\begin{tabular}{|l|l|}
\hline Density $\left[\mathrm{g} / \mathrm{cm}^{3}\right]$ & 0.97 \\
\hline Diameter $[\mu \mathrm{m}]$ & 18 \\
\hline Tensile strength $[\mathrm{MPa}]$ & 2500 \\
\hline Young's modulus $[\mathrm{GPa}]$ & 80 \\
\hline Strain capacity $[\%]$ & 4 \\
\hline
\end{tabular}

The SHCC and its constituent matrix were cast in prismatic molds with dimensions $100 \mathrm{~mm} \times$ $100 \mathrm{~mm} \times 400 \mathrm{~mm}$. At both ends of the prisms, slices with a thickness of $30 \mathrm{~mm}$ were cut to prevent the wall effect on the fiber orientation in the specimens. The cylindrical specimens were then core drilled in the longitudinal direction of the prisms.

Core-drilled specimens with a length of $40 \mathrm{~mm}$ and a diameter of $22 \mathrm{~mm}$ were used as the reference to study both the quasi-static and dynamic behavior of the SHCC and its constituent matrix; see Figure 1a. Five specimens were tested for each loading condition. In order to obtain the dynamic Young's modulus, three core-drilled specimens with a length of $100 \mathrm{~mm}$ were used. The long specimens were instrumented with four strain gauges, as shown in Figure 2. With a known distance between the two longitudinally positioned strain gauges, the wave velocity and the dynamic Young's modulus was calculated. All the experiments were performed at an age of 28 days. 




a)

b)



c)

Figure 1. a) Reference specimen geometry used for dynamic and quasi-static tests on SHCC and plain matrix, b) geometry of the short SHCC specimens, and c) geometry of the annular SHCC specimens.

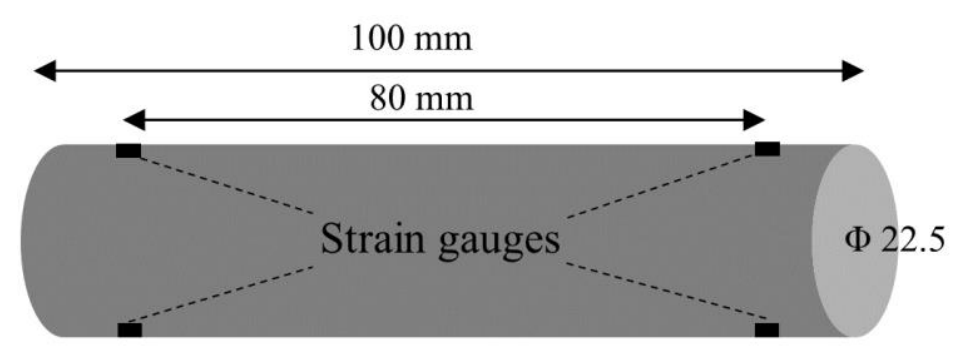

Figure 2. Dimensions of the long specimen used for deriving Young's modulus, and positions of the strain gauges attached to the specimen.

In addition to the reference geometry, specimens with a shorter length and annular specimens were produced. The shorter specimens had a length of $20 \mathrm{~mm}$ and the same diameter as the references; see Figure 1b. The annular specimens had the same length as the reference geometry, with outer and inner diameters of $22 \mathrm{~mm}$ and $10 \mathrm{~mm}$, respectively; see Figure 1c. These specimens were tested on the split Hopkinson bar to study the mechanisms behind the influence of structural inertia.

\subsection{Quasi-static tensile test configuration}

The quasi-static tension tests were performed using a Zwick 1445 testing machine in a deformation-controlled regime at a cross-head displacement rate of $0.04 \mathrm{~mm} / \mathrm{s}$. The strain rate in the specimens before cracking was $0.001 \mathrm{~s}^{-1}$. For these experiments, two metallic cylinders were glued at both ends of the specimen as extensions. These cylinders were then glued inside two rings which could be attached to the testing machine; see Figure 3. The use of extension cylinders at the two ends of each specimen prevented direct embedment of the specimen's ends in the glue. Thus, the shear stress created by contact between the glue and circumference area of the specimen is eliminated. As a result, it could be assumed that the boundary conditions of 
the specimens in quasi-static experiments were identical to those performed in the SHTB. A fast hardening, bi-component glue consisting of a fluid component DEGADUR®1801 and a powder component DEGADUR® $7742 \mathrm{~F}(\mathrm{~N})$, both produced by Evonik Röhm GmbH, was used for gluing the extension cylinders inside the steel rings.

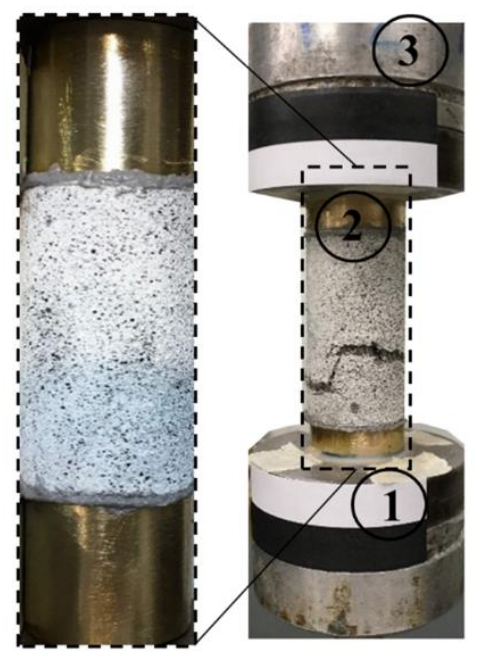

1. Fixing ring and the target attached on it for optical measurement

2. Sample glued to the extension cylinder

3. Connection to the testing frame

Figure 3. Setup used for quasi-static tension tests.

\subsection{Split Hopkinson tension bar}

The gravitational split Hopkinson tension bar (SHTB) presented in Figure 4 was used to perform impact tensile experiments. A short description of the setup is provided here, while a more detailed one can be found in [6]. The SHTB consists of an aluminum input bar at the bottom with a length of $1.84 \mathrm{~m}$ and a brass transmitter bar at the top with a length of $2 \mathrm{~m}$. The transmitter bar is rigidly attached at its top end to an aluminum frame. The input bar is attached to a flange which is hit by the impactor. The input and transmitter bars are laterally fixed at the position of the horizontal beams placed on the main frame of the setup; see Figure 4. At the fixing points, plastic bushings with a low coefficient of friction are used to keep the bars in position. The total height of the setup is $4.2 \mathrm{~m}$, which is restricted by the height of the laboratory room in which it was installed.

The setup was developed for impact tensile tests on strain-hardening, cement-based materials such as SHCC and TRC [6], [20]. The multiple cracking of these composites dictates a long representative specimen size, thus, introducing a challenge in reaching equilibrium in the specimen. Moreover, the long and ductile specimens require a long input wave to capture the stress-strain relation up to failure localization.

Based on the average crack spacing of the SHCC under investigation and a series of calibration experiments, a length of $40 \mathrm{~mm}$ was selected for the specimens. A transmitter bar having a high 
impedance mismatch with the specimen and an input wave with a long rise time facilitated reaching dynamic equilibrium in such long specimens. The use of a transmitter bar made of brass, which has a high impedance mismatch with the specimen, leads to a faster rise of stress at the specimen's end connected to the transmitter bar [21], [22]. Therefore, stress equilibrium in the specimen can be reached faster than is the case when both bars are made of aluminum. Analytical and experimental studies on the use of aluminum input bar and brass transmitter bar can be found in previous research performed using the setup [6].

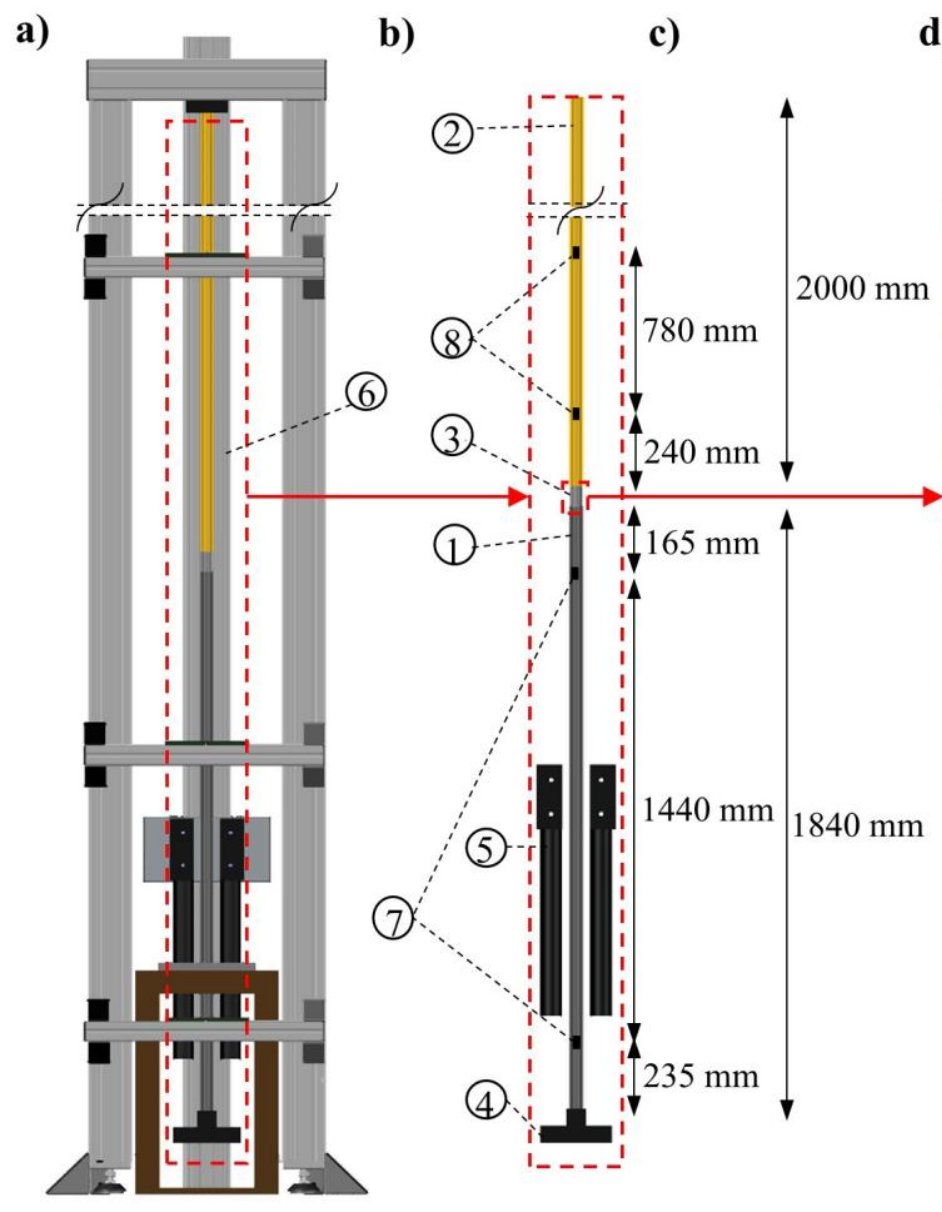

d)

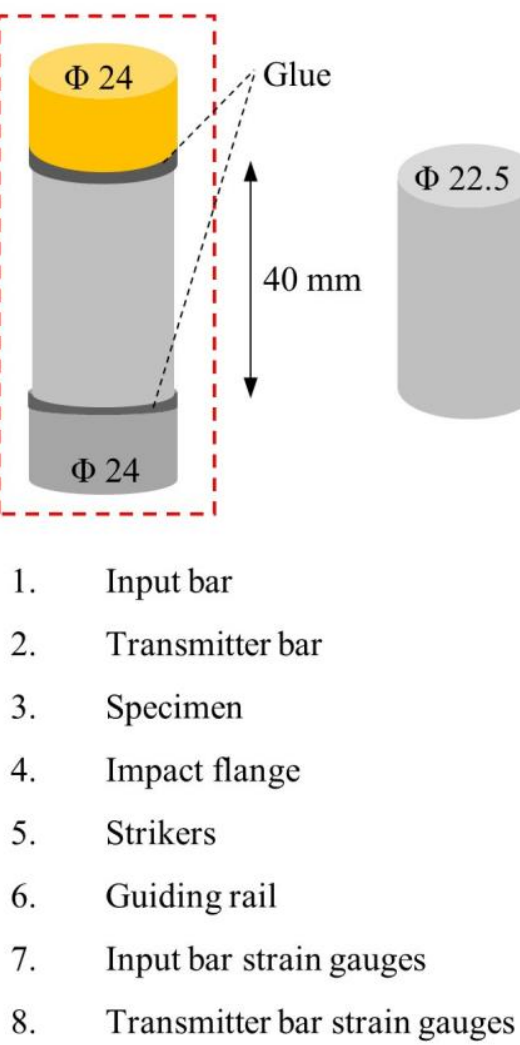

Figure 4. The gravity-driven split Hopkinson tension bar: a) schematic view of the setup; b) main components; c) position of the strain gauges and length of the bars; d) dimensions of the sample and its attachment to the bars.

The rigid body motion of the impact flange, made of stainless steel, is used to generate a sufficiently long input wave with a long rise time [23], [24]. As a result of the high wave propagation velocity in stainless steel and the high structural rigidity of the flange, the vibration of the flange is negligible compared to its translational movement. In this way, rigid body motion of the flange occurs under the direct action of the strikers, i.e., impulse-response rigidity or force-response rigidity [23]. As a consequence, the length of the resulting input wave is more 
than double the strikers' lengths [6], which enables the use of relatively short strikers. Moreover, since the input wave is not generated by direct contact of the striker on the input bar, a long rise time for the input wave was obtained. The striker device in the SHTB consisted of two parallel $0.4 \mathrm{~m}$-long steel bars tightened to two steel blocks and positioned on both sides of the input and transmitter bars. The steel blocks were connected by an aluminum plate. The total weight of the striker assembly was $30 \mathrm{~kg}$ and could be released from the selected drop height.

Figure 5a shows the input, reflected, and transmitted waves for a SHCC specimen. The input pulse presented in this figure corresponded to a drop height of $1 \mathrm{~m}$. This pulse was able to ensure a $3.2 \mathrm{~mm}$ deformation in a 40-mm long SHCC specimen at a maximum deformation rate of $8 \mathrm{~m} / \mathrm{s}$. The $3.2 \mathrm{~mm}$ deformation applied by one wave passage was enough to achieve failure (complete separation) in the $40 \mathrm{~mm}$-long SHCC specimens with a strain capacity of up to $8 \%$.
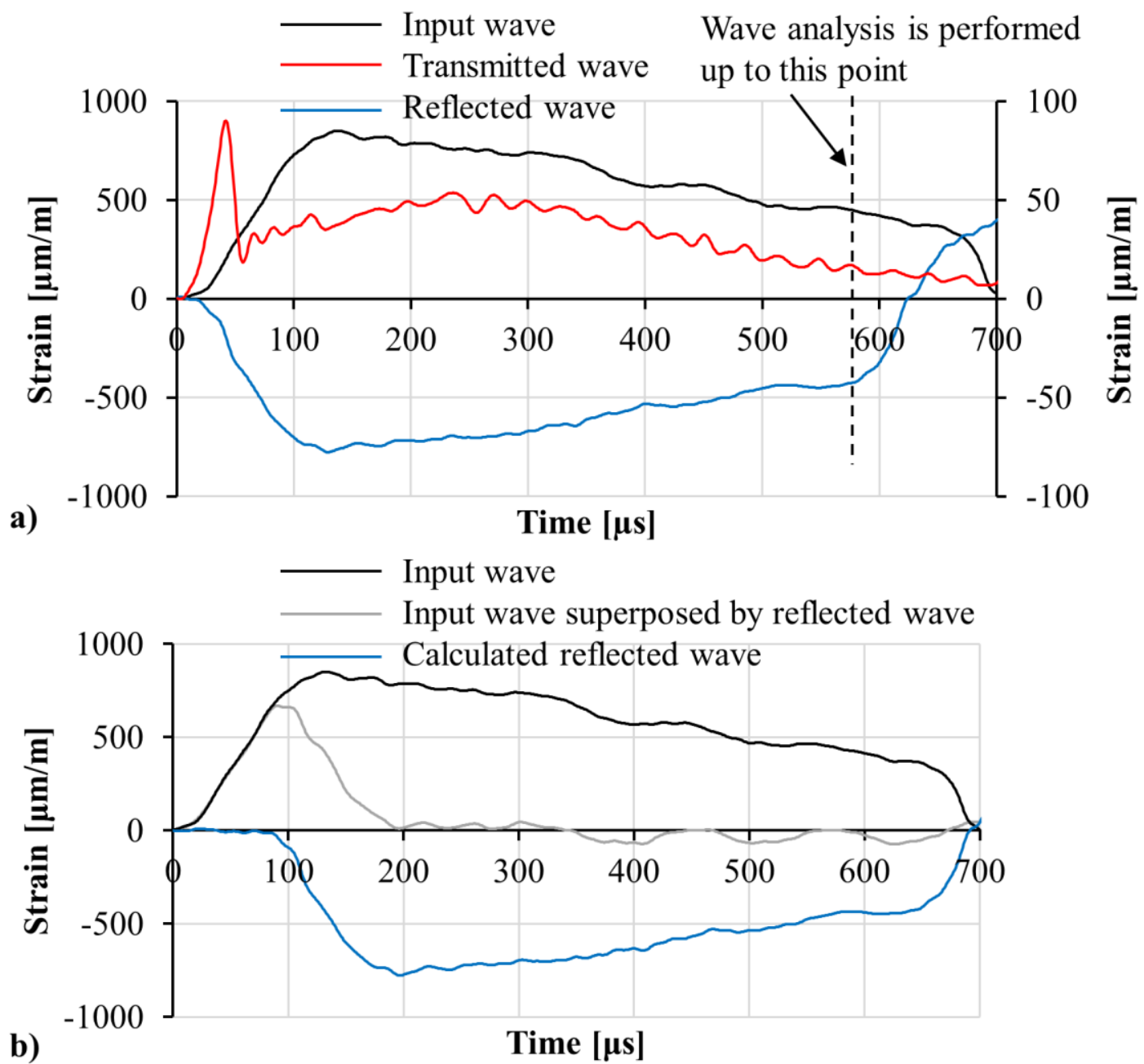

Figure 5. a) Example of the input, reflected, and transmitted waves for a SHCC specimen. The transmitted wave is shown on the secondary vertical axis; b) waves recorded on the bottom end and top end of the input bar, and the derived reflected wave. 
Recording a long input wave requires a sufficiently long input bar. Conventionally in split Hopkinson bars, the input bar is longer than the input wave. So, by using only one measurement point in the middle of the input bar, both input and reflected waves can be recorded. The use of a single measurement point in the middle of the input bar is only applicable if the input wave and the reflected wave can be recorded separately in time without being superposed by one another [25]. This condition is not fulfilled in the designed gravity-driven SHTB since the input wave is generated by the rigid body movement of the impact flange, which continues its movement even after losing contact with the striker [6]. This issue can be solved by using two measurement points on the input bar, which has the advantage that disregarding the length of the input wave both input and reflected waves can be obtained for any arbitrary measurement duration [26][28]. In this method, the strain gauges must have at least a distance of five times the diameter of the bar to the bar's end to ensure 1-D wave propagation [29], [30]. Moreover, the influence of wave dispersion becomes more prominent when this method is used for longer measurement times [28], which was not the case in this investigation. In the split Hopkinson tension bar developed, the input wave recorded by the strain gauges at the bottom side of the input bar was shifted on the time axis to the recording of the top strain gauges, then it was subtracted from the wave recorded at that point; see Figure 5b. The result is the wave reflected from the specimen. The reflected wave determined was only valid for $0.57 \mathrm{~ms}$, which is equal to twice the time needed for the wave to travel between the top and bottom strain gauges. The input wave and the derived reflected wave were then shifted on the time axis to the bottom side of the specimen in contact with the input bar and were used for the conventional split Hopkinson bar wave analysis.

All strain gauges were sampled at a rate of $1 \mathrm{MHz}$ and were subsequently filtered with a zerophase, $60 \mathrm{kHz}$ low-pass filter to cancel the electrical noise. A Fast Fourier Transform (FFT) analysis was performed on the recorded waves to check the suitability of the selected low-pass filter. Figure 6 shows that the contribution of frequencies higher than $20 \mathrm{kHz}$ is close to zero. Thus, the selected $60 \mathrm{kHz}$ low-pass filter eliminated the noise only. It should be noted that the FFT analysis was performed on the signals recorded for $0.7 \mathrm{~ms}$ at a sampling rate of $1 \mathrm{MHz}$. Thus, the frequency resolution of the FFT results was limited to $1428 \mathrm{~Hz}$, which was sampling frequency divided by the number of samples recorded in $0.7 \mathrm{~ms}$. 


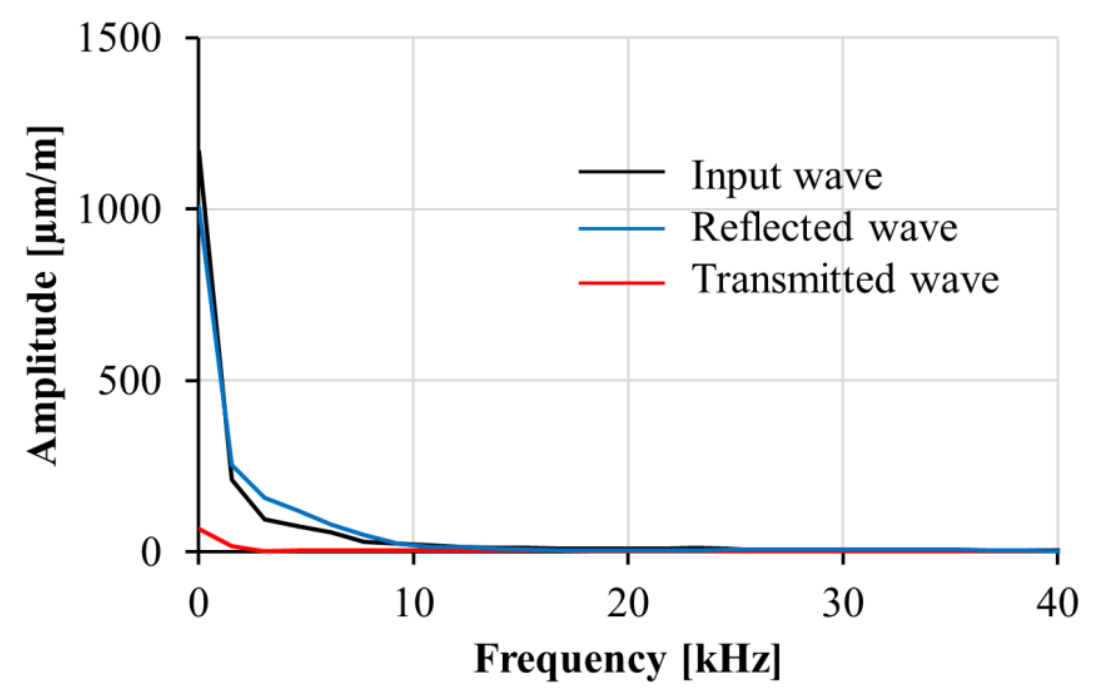

Figure 6. Fast Fourier Transform (FFT) of the recorded waves in an impact tensile test on a cylindrical SHCC specimen.

The wave analysis was performed according to the theory of one-dimensional wave propagation [31]. The stresses at both ends of the specimen were compared to evaluate dynamic stress equilibrium. Their average was considered for evaluation of the material properties. Eqs. 1 and 2 are used here in calculating the forces at each end of the specimen based on the input, reflected, and transmitted waves, i.e. $\varepsilon_{I n}, \varepsilon_{R e}$, and $\varepsilon_{T r}$, respectively. The subscripts $i$ and $t$ are attributed to the input and transmitter bar, respectively.

$F_{i}(\mathrm{t})=E_{i} A_{i}\left(\varepsilon_{I n}(\mathrm{t})+\varepsilon_{R e}(\mathrm{t})\right)$

$F_{t}(\mathrm{t})=E_{t} A_{t} \varepsilon_{T r}(\mathrm{t})$

The displacements at the ends of the specimen are calculated based on Eqs. 3 and 4, where $C_{i}$ and $C_{t}$ are elastic wave velocities in the input and transmitter bars, respectively.

$\delta_{i}(\mathrm{t})=C_{i} \int_{0}^{t}\left(\varepsilon_{I n}(\mathrm{t})-\varepsilon_{R e}(\mathrm{t})\right) d t$

$\delta_{t}(\mathrm{t})=C_{t} \int_{0}^{t} \varepsilon_{T r}(\mathrm{t}) d t$

The stress in the specimen is calculated using Eq. 5, where $A_{s}$ is the cross-sectional area of the specimen.

$\sigma(\mathrm{t})=\frac{F_{i}(\mathrm{t})+F_{t}(\mathrm{t})}{2 A_{s}}$

The strain and strain rate in the specimen is estimated using Eqs. 6 and 7, respectively, where $L_{S}$ is the initial length of the specimen.

$\varepsilon(\mathrm{t})=\frac{\delta_{i}(\mathrm{t})-\delta_{t}(\mathrm{t})}{L_{s}}$ 
$\dot{\varepsilon}(\mathrm{t})=\frac{\left(\varepsilon_{I n}(\mathrm{t})-\varepsilon_{R e}(\mathrm{t})\right) C_{I}-\varepsilon_{T r}(\mathrm{t}) C_{T}}{L_{S}}$

Note that since two measurement points on the input and transmitter bars were used, the elastic wave velocity and Young's modulus of the bars were derived directly. The correct measurement of these parameters is of great importance in shifting the signals towards the specimens' ends as well as in deriving the stress in the specimen.

The setup was also equipped with two high-speed cameras of type SA-X2 produced by Photron ${ }^{\circledR}$. In the conducted experimental series, the cameras captured 160000 frames per second with a resolution of $120 \times 512$ pixels. The captured frames were then used for a 3D DIC analysis. Aramis software produced by GOM GmbH was used for performing the DIC.

\section{Experimental program at the microscale}

\subsection{Specimen production}

Specimens consisting of a single HDPE fiber embedded in the normal-strength matrix block were produced using the mold shown in Figure 7. For the production of specimens, the fibers were first fixed in the grooves designed in the mold. The cement-based matrix was then injected to fill the grooves [32]. The widths of the grooves can be adjusted using spacer plates with different thicknesses according to the required embedment length. In the experiments presented in this study, embedded length was set to $4 \mathrm{~mm}$.


Figure 7. The mold used for the production of the fiber pullout specimens.

\subsection{Quasi-static fiber pullout test setup}

Quasi-static experiments were performed in a Zwick 1445 universal testing machine with a pullout speed of $0.001 \mathrm{~mm} / \mathrm{s}$. The pullout force was measured using a $10 \mathrm{~N}$ load cell, and the 
displacement was acquired as the cross-head displacement of the testing machine. The setup of the quasi-static, single-fiber pullout experiments can be found in more detail in [32].

\subsection{Miniaturized split Hopkinson tension bar}

A mini-Hopkinson tension bar was used to investigate the influence of pullout speed on the crack-bridging behavior of the fibers. The setup is capable of performing single fiber pullout experiments at displacement rates up to $2000 \mathrm{~mm} / \mathrm{s}$. This speed was selected based on the crack opening speed, which was observed using DIC on the SHCC specimens tested in the SHTB. Figure 8 shows the schematic view of the mini-Hopkinson tension bar developed.

In this setup an input bar with a diameter of $4 \mathrm{~mm}$ and a length of $2 \mathrm{~m}$ was used. A cylindrical flange was screwed on the right end of the input bar. A hollow cylinder made of steel, with a length of $1 \mathrm{~m}$ and an outer and inner diameter of $10 \mathrm{~mm}$ and $7 \mathrm{~mm}$, respectively, was used as a striker. The striker was accelerated using a spring. Once the striker was released, it moved around the input bar and hit the impact flange. The input bar and the impact flange were supported laterally to prevent their free movement during the test. Moreover, the striker was placed on two roller supports to ensure its smooth movement and to prevent its contact to the input bar once it was shot toward the impact flange. The supports of the input bar and the supporting rollers of the striker are shown in Figure $\mathbf{8}$ in blue color. Since the setup was designed for fiber pullout tests and single fiber tension tests in which the measured forces are typically close to $1 \mathrm{~N}$, the typical wave analysis of the split Hopkinson bar based on the recorded strain waves on the bars was not feasible [33], [34]. Thus, the transmitter bar was replaced with a load-cell to solve this issue. A load cell of type Kistler 9205 with a capacity of $50 \mathrm{~N}$ was placed on the block shown in Figure 8 in red color on the left side of the setup. Furthermore, the pullout displacement was measured by an optical extensometer of type 200XR produced by H.-D. Rudolph GmbH. The target for the optical extensometer was attached to the adapter where the matrix block was fixed; see Figure 8. 
a)

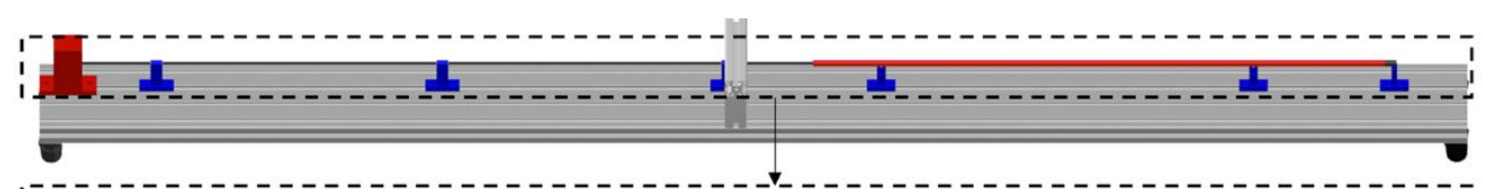

b)

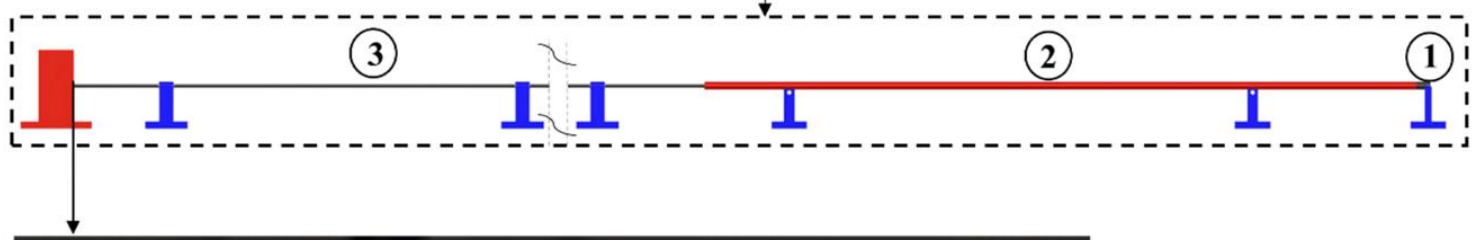
1. Impact flange
2. Tubular striker
3. Input bar
5. Specimen glued on an adapter with a target for optical measurement

Figure 8. a) Schematic view of the mini-Hopkinson tension bar developed for high-speed micromechanical investigations, and b) a fiber pullout specimen tested in the setup.

\section{Results and discussion}

\subsection{Quasi-static tensile experiments}

\subsubsection{Results of non-reinforced matrix}

An investigation into the quasi-static behavior of the non-reinforced matrix was performed. The cementitious matrix was tested under identical loading scenarios with identical specimen geometries as the SHCC. The mechanical properties obtained are given in Table 3. In the quasistatic loading regime, the tensile strength of the matrix was 3.3 MPa. The tensile Young's modulus was derived from the stress-strain relationships in the range between $30 \%$ and $60 \%$ of the peak tensile stress [35]. The average Young's modulus of the matrix was $17.8 \mathrm{GPa}$. The low value of Young's modulus is a result of the high binder content, specifically that of fly ash; see

\section{Table 1.}

\subsubsection{Results of SHCC}

The tensile behavior of SHCC can be characterized by the first crack stress, tensile strength, and strain at peak tensile stress, that is, strain capacity. First crack stress depends predominantly on the tensile strength of the matrix, but on the bond between fibers and matrix as well. The hydrophobicity of the HDPE fibers ensures mechanical interaction with the cementitious matrices with no chemical bond [7]. Additionally, the relatively high porosity and considerable inhomogeneity of the normal-strength matrix at micro-level further reduces fiber-matrix bond 
strength. Despite all that, the fibers have a positive effect on the first-crack stress of SHCC when compared to the tensile strength of the corresponding non-reinforced cementitious matrix, showing an enhancement from 3.3 MPa to 4.5 MPa; see Table 3. This can be traced back to the fact that the well-distributed microfibers hinder microdefects' development to larger cracks. Figure 9 shows the obtained stress-strain relations.

The weak bond between fibers and matrix also leads to limited crack-bridging capacity in the composite. As a result, the tensile strength of the SHCC investigated is below the first-crack stress obtained. Moreover, limited strain hardening behavior is observed. The strain-hardening branch of the stress-strain curve was accompanied by poor multiple cracking, which is restricted to the region where localization of failure occurred. Figure $\mathbf{1 0}$ shows the crack pattern on a SHCC specimen tested under quasi-static tensile load. The crack pattern was obtained by DIC.

Table 3. Mechanical properties of the non-reinforced cementitious matrix and SHCC under quasi-static tensile loading; standard deviations are given in parentheses.

\begin{tabular}{|c|c|}
\hline \multicolumn{2}{|l|}{ Non-reinforced matrix } \\
\hline Tensile strength [MPa] & $3.3(0.2)$ \\
\hline Young's modulus [GPa] & $17.8(2.7)$ \\
\hline \multicolumn{2}{|l|}{ SHCC } \\
\hline First-crack stress [MPa] & $4.5(0.6)$ \\
\hline Tensile strength ${ }^{1}[\mathrm{MPa}]$ & $3.6(1.1)$ \\
\hline Young's modulus [GPa] & $21.3(4.2)$ \\
\hline Ultimate $\operatorname{strain}^{1}[\%]$ & $0.6(0.2)$ \\
\hline Work-to-fracture ${ }^{1}\left[\mathrm{~kJ} / \mathrm{m}^{3}\right]$ & $19.7(2.8)$ \\
\hline
\end{tabular}

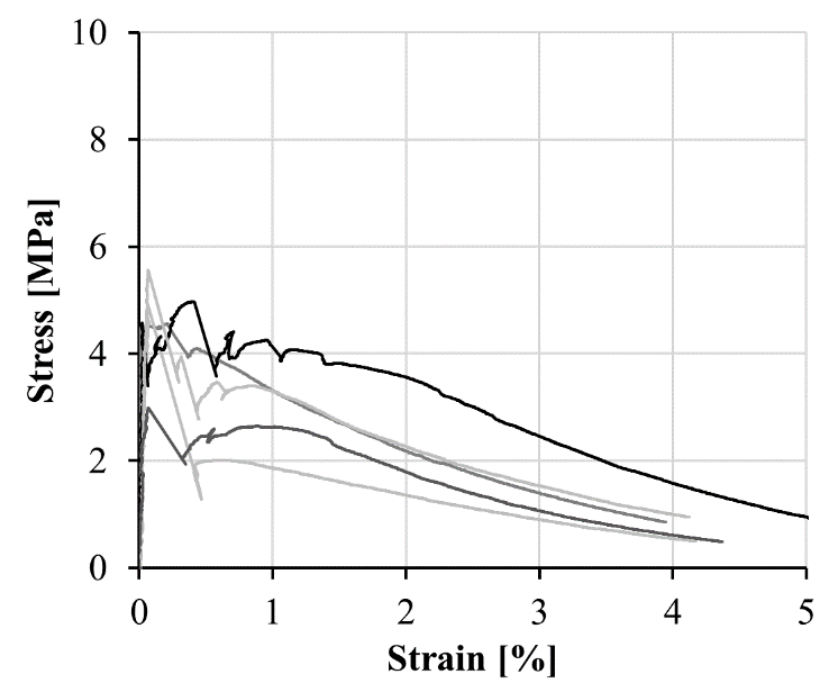

Figure 9. Stress-strain behavior of the SHCC under quasi-static tensile loading. 


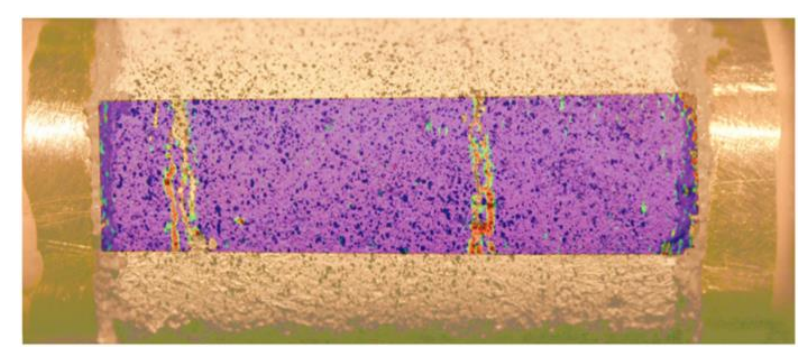

Figure 10. Cracking pattern of SHCC specimen under quasi-static tensile loading; image obtained from DIC analysis.

\subsection{Impact tensile experiments}

\subsubsection{Results of non-reinforced matrix under impact tensile load}

An example of the stress histories at the specimen-input bar and specimen-transmitter bar interfaces for a specimen made of plain matrix is shown in Figure 11. Eq. 1 and 2 were used to find stresses at the two ends of the specimen connected to the input and transmitter bars, respectively. After the initial non-equilibrium state in the specimen, the stresses at the specimens' ends match, and stress equilibrium is reached before failure. The equilibrium in the specimen ensures an accurate derivation of the stress and strain values based on the wave analysis. The contribution of inertial forces to the derived stress histories at the two ends of the specimen is discussed in section 4.2.3.

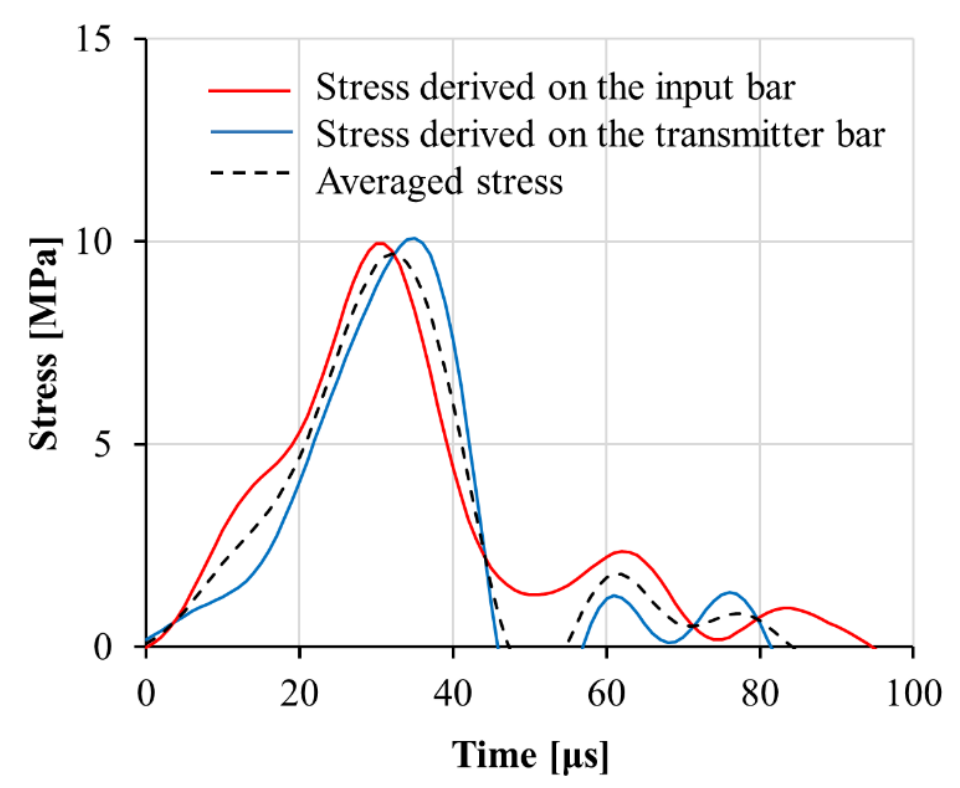

Figure 11. Stress-time histories calculated at the specimen-input bar interface and specimentransmitter bar interface for a non-reinforced matrix specimen.

The stress-strain diagrams of all the tested specimens are presented in Figure 12. The full-field strain evaluation done with DIC is also presented in the figure for a representative test to show 
the fracture mechanism; the corresponding stress-strain curve is the thicker black line in the diagram. For the frames presented, the associated stress and strain values are also given. The frames provided in Figure 12b were captured every $6.25 \mu \mathrm{s}$, and can be depicted as: I) the frame immediately prior to peak stress, showing minor damage in the form of microcracks, which appear as a bright blue region in the strain field; II) the frame immediately after peak stress, showing the extensive growth of microcracks in the entire specimen; and III) a frame taken on the descending branch of the curve showing a crack in the middle of the specimen. The time given in Figure 12b for each frame is the time passed from the moment the input wave reached the specimen.

a)



b)

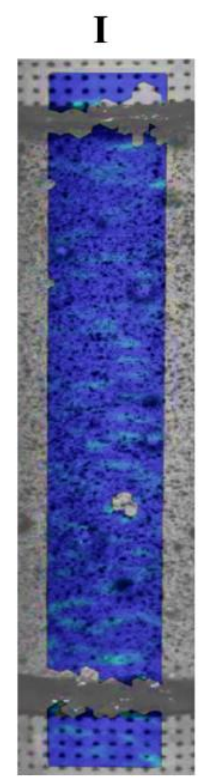

$\sigma=4.9 \mathrm{MPa}$

$\varepsilon=0.02 \%$

$\mathrm{T}=43.75 \mu \mathrm{s}$
Strain $[\%]$

II



$\sigma=10.9 \mathrm{MPa}$

$\varepsilon=0.05 \%$

$\mathrm{T}=56.25 \mu \mathrm{s}$
III

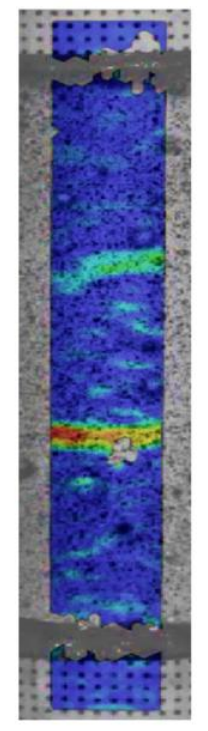

$\sigma=7.5 \mathrm{MPa}$

$\varepsilon=0.08 \%$

$\mathrm{T}=62.25 \mu \mathrm{s}$ 
Figure 12. a) Stress-strain curves of the non-reinforced cementitious matrix specimens tested in SHTB, and b) a representative DIC sequence showing local strains for various stress and average strain levels corresponding to the black stress-strain curve.

The cement-based matrix shows an apparent increase in the tensile strength under dynamic loading, with a dynamic increase factor (DIF) of 3.0; see Table 4 and Table 5. Note that for a specific material parameter, DIF is the ratio of the value obtained in a dynamic test to the quasistatic value. DIF is used in this study to quantify the change in material performance due to dynamic loading.

The following mechanisms are responsible for the increase of the tensile strength of cementbased matrices at strain rates higher than $1 \mathrm{~s}^{-1}$ : a) viscous behavior of the bulk material related to the influence of water trapped in the concrete capillary system [36], b) crack-tip inertia, which acts against an arbitrary increase in the crack-propagation speed when increasing the loading speed [13], [14], and c) structural level inertia, which is related to the mass and geometry of the specimen as well as the acceleration applied to the specimen [18]. While effects caused by the viscosity of the cement-based matrix and the micro-level inertia can be interpreted as genuine material rate-dependent behavior. The influence of structural-inertia should be differentiated from the material behavior [14]. Thus, a detailed discussion of structural-inertia is provided below in this section to extract the genuine rate-sensitivity of the material from the experimental results obtained on the SHTB.

Young's modulus of the matrix was found based on Eq. 8, using the time difference between the rising points of the recorded signals at the two measurement positions with a distance of 80 $\mathrm{mm}$ on the $100 \mathrm{~mm}$-long specimens; see Figure 2. A moderate increase in Young's modulus of the cement-based matrix under dynamic loading was observed in the present investigation; see Table 5.

$E_{D y n}=C^{2} \rho$

\subsubsection{Results of SHCC under impact tensile loading}

The stress-strain relations of the SHCC presented in Figure 13a show a peak in stress value at the beginning. This pronounced stress peak corresponds to first-crack stress and appears as a result of the apparent higher tensile strength of the cement-based matrix at the high strain rate, as discussed based on the results of non-reinforced matrix. 




a)

Strain [\%]



\section{II}

III

IV

V
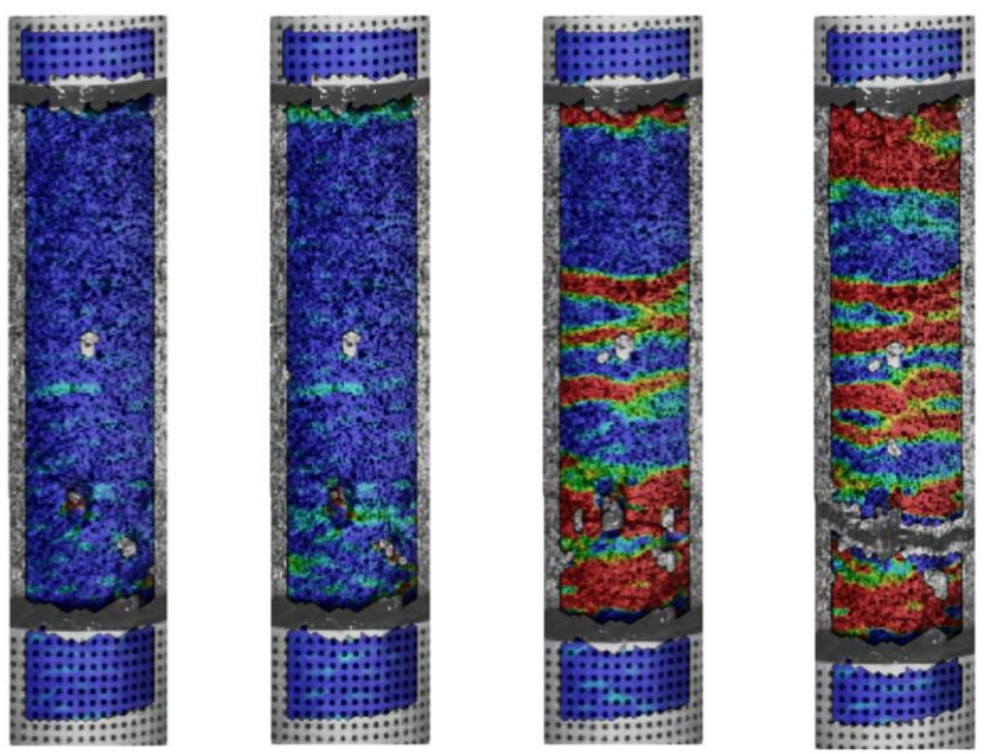

$$
\begin{array}{ccccc}
\sigma=2.3 \mathrm{MPa} & \sigma=8.8 \mathrm{MPa} & \sigma=2.5 \mathrm{MPa} & \sigma=7.3 \mathrm{MPa} & \sigma=2.9 \mathrm{MPa} \\
\varepsilon=0.01 \% & \varepsilon=0.05 \% & \varepsilon=0.14 \% & \varepsilon=2.4 \% & \varepsilon=5.7 \%
\end{array}
$$

$\mathrm{T}=31.25 \mu \mathrm{s} \quad \mathrm{T}=56.25 \mu \mathrm{s} \quad \mathrm{T}=68.75 \mu \mathrm{s} \quad \mathrm{T}=206.25 \mu \mathrm{s} \quad \mathrm{T}=406.25 \mu \mathrm{s}$

Figure 13. a) Stress-strain curves of the SHCC specimens tested in SHTB; b) a representative DIC sequence, showing local strains for various stress and average strain levels corresponding to the thicker black stress-strain curve.

The SHCC specimens undergo multiple cracking. The stress waves created by the formation of multiple cracks also start to reverberate in the specimen. Thus, the uniformity of stress in the specimen is partially lost after the first crack. The stress values derived at both ends of a specimen provided in Figure 14 can show the non-uniform stress in the specimen after the first crack. Moreover, as multiple cracking occurs in the specimen, the wave propagation velocity decreases significantly. The reduction in wave velocity negatively influences re-establishing the equilibrium in the specimen after the first crack. 



Figure 14. Stress-time histories in an SHCC specimen derived in the aluminum input bar and brass transmitter bar.

The full-field strain measurement obtained by DIC was used to visualize damage and cracking in the specimens. Figure 13b presents frames related to the black stress-strain curve. Frames I and II show the strain field on the specimen before the stress peak and right after the stress peak, respectively. A comparison of the first two DIC frames presented for SHCC with those of the plain matrix reveals similar fracture processes. The first crack visible as a localized strain field always appears after the first stress peak. Before this point, the damage evolution in the form of microcracks occurs in the matrix. Moreover, the tensile strength of the matrix fits the first stress peak in the stress-strain relations of SHCC. These similarities in experimental data indicate that the stress peak is attributable to the first-crack stress of the matrix. Frame III, corresponding to $68.75 \mu \mathrm{s}$ after the start of loading, shows the specimen at the end of the stress drop after the first crack. The last two frames, IV and V, were captured at the end of the hardening branch and at the end of the softening branch, respectively.

Both the first stress peaks in the case of SHCC specimens and the maximum stress obtained for plain matrix specimens show the apparent increase in the tensile strength of the cement-based matrix, which is caused by structural inertia, in addition to the genuine rate-sensitivity. The slight difference in tensile strength of the matrix, $9.8 \mathrm{MPa}$, and the stress peak observed in SHCC specimens related to first crack stress, $10.3 \mathrm{MPa}$, can be a result of the fibers' acting against the propagation and the merging of microcracks; see Table 4. A similar trend was also observed in the quasi-static experiments; see Table 3.

First crack formation causes specimen relaxation and, in turn, dramatic stress reduction, which terminates as the increasing crack opening activates the crack-bridging action of the fibers. At this moment, stress rises again, and the multiple cracking stage starts. The cracks generated at 
this stage appear at significantly lower stress levels than the first crack stress. This behavior can be explained based on the fact that before the first crack, distributed damage occurs in the matrix; see frame II in Figure 13b. As a result, the subsequent cracks are formed in a partially damaged, thus a weaker matrix.

Additionally, the results of DIC indicate that the strain rate after the first crack is not uniform in the specimen [6]. The regions between the opened cracks experience considerably lower strain rates as compared to the global strain rate. In Figure $\mathbf{1 5 b}$ the four light gray curves show the strain rate in the individual sections marked on the specimen. The frame showing the strain distribution in the specimen when the first macro crack forms is also provided. The strain rate in four distinct regions on the specimen and the average strain rate calculated based on the global deformation rate applied to the specimen can be compared. At the beginning of the test and before the formation of the first macro-crack, i.e. up to $50 \mu \mathrm{s}$, the strain rate in the small selected regions matches the average. As the first macro-crack is formed, the strain rate in the uncracked regions decreases to values close to zero. At the same time the local strain rate in two of the selected regions rises to values higher than the average due to the generation of cracks in these regions of the specimen. As a result, in the uncracked regions, the previously discussed mechanisms responsible for the increase in the tensile strength of the cement-based matrix cannot be activated to the extent to which they had been before cracking. Thus, the dynamic increase factor of the matrix cracking stress obtained in the multiple cracking phase $(\mathrm{DIF}=1.8)$ is lower than that of first-crack stress $(\mathrm{DIF}=2.3)$; see Table 5.

The frames used for DIC were captured every $6.25 \mu$ s, resulting in approximately one frame per reflection of the wave at the ends of the specimen. Thus, the strain field obtained by DIC can be used as an alternative method to examine the uniformity of strain in the specimens tested in SHTB. In Figure 15a, the four light gray curves show the strain in the individual sections marked on the specimen. It can be observed that strains measured in the sections start to show significant discrepancies after the formation of the first macro-crack. Nevertheless, before the formation of the first crack in the matrix, the measured strains are reasonably close to each other. Indeed the standard deviation of the strains in the four sections is much smaller than their average, indicating an acceptable uniformity of strain distribution in the specimen loaded dynamically. The limitations of the DIC analysis based on the images taken by high-speed cameras should also be considered. The intrinsic noise of the measurement results in a non-zero standard deviation even before deforming the specimen. So, upon the start of loading, an increase in the standard deviation due to the non-uniform strain in the specimen is not observable. 




a) Time $[\mu \mathrm{s}]$
Strain and strain rate in four sections

Average strain and average strain rate in four sections

- Standard deviation of strain in four sections

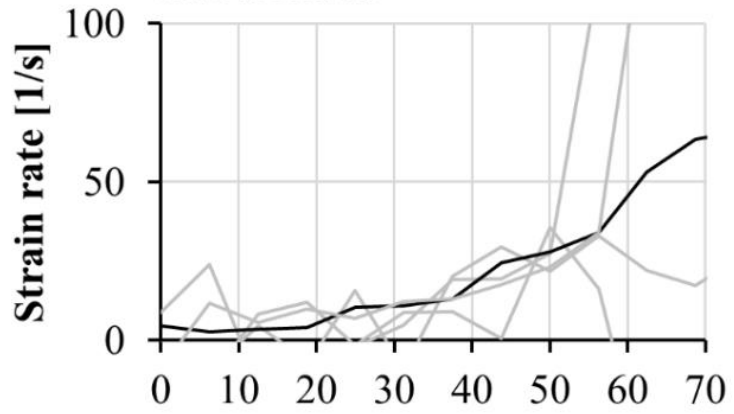

b)

Time $[\mu \mathrm{s}]$

Figure 15. Strain and strain rate history in four distinct regions of a representative specimen up to the first crack formation.

Once the results of impact tensile experiments are compared to those of quasi-static tests, DIF values of 3.5 and 5.9 are obtained for strain capacity and work-to-fracture, respectively. The enhancement in the performance of SHCC under impact loading emphasizes the importance of fiber-matrix bond and fiber strain-rate sensitivity on the performance of SHCC [5], [7]. At higher strain rates, tensile strength of the matrix, tensile strength of the fiber, and fiber-matrix bond strength change. The change of these material parameters due to dynamic loading leads to an alteration of the micromechanical conditions required for strain-hardening and multiple cracking [2]. The alteration of the materials parameters can be engineered in favor of the composite performance under impact loading.

An experimental program consisting of quasi-static and high-speed fiber pullout tests was performed using the mini-Hopkinson tension bar presented. The results of the micromechanical investigations under dynamic loading indicate that in the SHCC under investigation, the fibermatrix bond is strongly enhanced in comparison to the weak bond observed in the quasi-static regime. As a result, the fibers' crack-bridging capacity is also enhanced; see section 4.3 for a detailed discussion of the results. At the same time, the significant enhancement of the tensile strength of the matrix due to the high strain rate only occurs when the first crack is formed. The distributed damage of the matrix before the first macro-crack and the reduction of the local strain rate in the uncracked regions of the matrix allow for further multiple cracking at lower stress levels. The improved multiple-cracking behavior results in improved strain capacity and energy absorption compared to the quasi-static regime. 
Same as for the plain matrix, Young's modulus of the SHCC was calculated by measuring the wave propagation speed in specimens with a length of $100 \mathrm{~mm}$ instrumented with strain gauges as shown in Figure 2. A data recorder with a sampling frequency of $20 \mathrm{MHz}$ was used in these particular experiments to increase the temporal resolution of the signal recorded by the strain gauges and, as a result, the accuracy of the derived Young's modulus.

Table 4. Mechanical properties of the non-reinforced cementitious matrix and SHCC under quasi-static tensile loading; standard deviations are given in the parentheses.

\begin{tabular}{|c|c|}
\hline \multicolumn{2}{|l|}{ Non-reinforced matrix } \\
\hline Tensile strength [MPa] & $9.8(1.8)$ \\
\hline Young's modulus [GPa] & $23.7(4.8)$ \\
\hline \multicolumn{2}{|l|}{ SHCC } \\
\hline First-crack stress [MPa] & $10.3(0.9)$ \\
\hline Tensile strength ${ }^{1}[\mathrm{MPa}]$ & $6.9(0.7)$ \\
\hline Young's modulus [GPa] & $25.9(2.1)$ \\
\hline  & $2.1(0.9)$ \\
\hline Work-to-fracture ${ }^{1}\left[\mathrm{~kJ} / \mathrm{m}^{3}\right]$ & $117.4(19.1)$ \\
\hline
\end{tabular}

Table 5. DIF of the mechanical properties for the investigated plain matrix and SHCC.

\begin{tabular}{|l|l|}
\hline \multicolumn{2}{|l|}{ Non-reinforced matrix } \\
\hline DIF of tensile strength & 3.0 \\
\hline DIF of Young's modulus & 1.3 \\
\hline SHCC & 2.3 \\
\hline DIF of first-crack stress & 1.8 \\
\hline DIF of tensile strength & 1.2 \\
\hline DIF of Young's modulus & 3.5 \\
\hline DIF of ultimate strain & 5.9 \\
\hline DIF of work-to-fracture & \\
\hline${ }^{1}$ Obtained at the maximum stress after the first crack. \\
\hline
\end{tabular}

\subsubsection{Influence of inertia}

The tensile strength of the plain cement-based matrix and the first-crack stress of the SHCC are considerably higher in the impact tests as compared to the quasi-static tests; see Table 5. The absence of such a dramatic increase in the tensile strength of the matrix in the multiple cracking phase was explained. However, further investigations were performed to extract the genuine rate sensitivity from the apparent increase of the matrix tensile strength caused by structural inertia. In the range of strain rate obtained in the SHTB tests, reaching $50 \mathrm{~s}^{-1}$ at the moment that the first crack is formed, the predominant factors influencing the results are structural inertia and crack-tip inertia rather than the viscosity of the cement-based matrix [36], [37]. 
Upon propagation of the crack, the material around the crack tip must move to enable crack opening. The movement of the material around the crack tip creates an acceleration field in the adjacent mass. The acceleration causes inertial forces that tend to prevent crack propagation. The counteracting influence of inertia against the opening of cracks limits crack propagation speed [15]. Theoretically, neglecting inertial forces, crack propagation speed should reach Rayleigh wave speed, which is the propagation speed of the waves traveling on the surface of a solid. However, once inertial forces are considered, crack propagation speed proves to be significantly lower than the Rayleigh wave speed [15], [35], [38]. The limited crack propagation speed and the very high loading rate in the split Hopkinson tension bar also lead to an increase in the tensile strength of the quasi-brittle specimen [39]. The specimen does not exhibit damage as rapidly as it is loaded, so it can bear higher loads. It should be noted that at the macroscale, crack-tip inertia and viscosity of the matrix can be considered as genuine material rate sensitivity. Contrarily, structural inertia should be extracted from the experimental results, since it cannot be attributed to material behavior.

\section{Structural inertia}

Structural inertia is related to the acceleration of the bulk mass of the specimen. The sources of the acceleration are the loading applied to the specimen as well as the softening and damage of the material, which occurs under loading [18]. The inertial force generated in the specimen affects the stress derived at the two ends of the specimen based on the recorded waves in SHTB. Initially, the simplified case in which the specimen deforms elastically and acceleration is induced only by the loading wave was considered. With the assumption that the specimen deforms elastically under the applied load, several analytical models are available to predict the contribution of inertia and to optimize the geometry of the specimen [40]. Generally, two approaches are used in developing such analytical models: the energy-based approach and the stress-based approach. While the energy-based approach uses the energy balance equation in the specimen, the stress-based approach uses the stress-field in the specimen to derive the contribution of inertia. These models consider both tractions and velocities applied on the two ends of the specimen, as presented schematically in Figure 16. Among all, the models developed by Gorham [41] and by Forrestal [42] represent well established energy-based and stress-based models, respectively. Moreover, it has been proved that the results obtained using the two approaches are consistent [43]. The models indicate that inertial forces are a function of strain rate and strain acceleration in the specimen and that the inertial forces generated are controlled by the length and diameter of the specimens. Indeed, the mechanism assumed for the 
contribution of inertia in these simplified models suggests that the axial and radial acceleration can be reduced by reducing the length and diameter of the specimen, respectively.

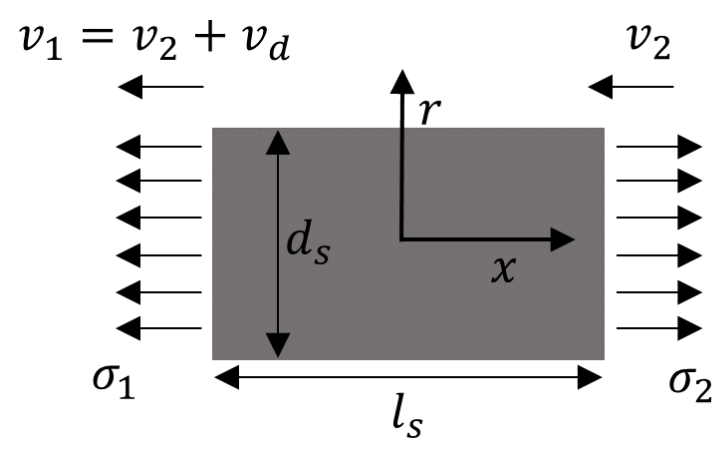

Figure 16. Schematic view of the velocities and tractions applied to the ends of a specimen that deforms elastically in a SHTB. Left side is connected to the input bar and right side is connected to the transmitter bar.

To scrutinize the applicability of such models in predicting the influence of structural inertia on the tensile strength of the cement-based matrix obtained in SHTB, experiments on SHCC specimens with various geometries were performed. The first crack stress of SHCC is directly related to the rate-dependant behavior of the cement-based matrix. The specimens were made of the same SHCC as that presented in Table 2. Shorter specimens with a length of $20 \mathrm{~mm}$ and annular specimens with a 10-mm opening were tested in the same SHTB; see Figure 1b and Figure 1c. As mentioned previously, the magnitude of the first stress peak in the stress-strain diagrams of SHCC specimens is directly related to the tensile strength of the cement-based matrix. By having a similar strain rate and strain acceleration in the tests, the length and the diameter of the specimen are the only variables influencing the contribution of inertia. Therefore, experiments on the specimens with different geometry can reveal the suitability of the assumed mechanisms for capturing the influence of structural inertia on the apparent increase in the tensile strength of the matrix.

The results of the impact tensile tests on shorter SHCC specimens are provided in Figure 17. The reduction of the specimen length from $40 \mathrm{~mm}$ to $20 \mathrm{~mm}$ should considerably decrease the inertia contribution to the stress calculated based on the wave analysis [41]. However, the obtained stress-strain relations for shorter specimens show almost no difference in the case of the first stress peak. The apparent increase in the tensile strength of the cement-based matrix is still observable to the extent it was for the long specimens, as shown in Figure 15. Note that the tensile impact experiments on shorter specimens were performed using an adjusted input wave, which resulted in a similar strain rate and strain acceleration history in the specimen. The 
releasing height of the striker was reduced to $0.4 \mathrm{~m}$ to reduce the amplitude of the input wave and achieve a strain rate history similar to that of the long specimens.

The results of the tests performed on annular specimens with a length of $40 \mathrm{~mm}$ using the SHTB are provided in Figure 18. According to the both models developed by Gorham and Forrestal [41], [42], the contribution of inertia also depends on the diameter of the cylindrical specimen. Thus, by taking out the inner core of the specimen, the contribution of inertia should decrease. Indeed, by creating an opening with a diameter of $10 \mathrm{~mm}$ in the center of the cylindrical specimen, the mass that is radially accelerated is considerably decreased. Thus, the influence of radial inertia should decrease [11]. Moreover, it is proved that the inertia-induced radial confinement decreases with an increasing distance from the center of a cylindrical specimen [42]. Therefore, by taking a part of the material from the inner core of the specimen, the portion of the specimen that is subject to the confinement is reduced. So, the influence of inertiainduced confinement on the obtained tensile strength of the matrix is also indirectly examined in these experiments. Remarkably, the results of the annular specimens also showed no noticeable change in the first stress peak, thus representing the tensile strength of the cementbased matrix.

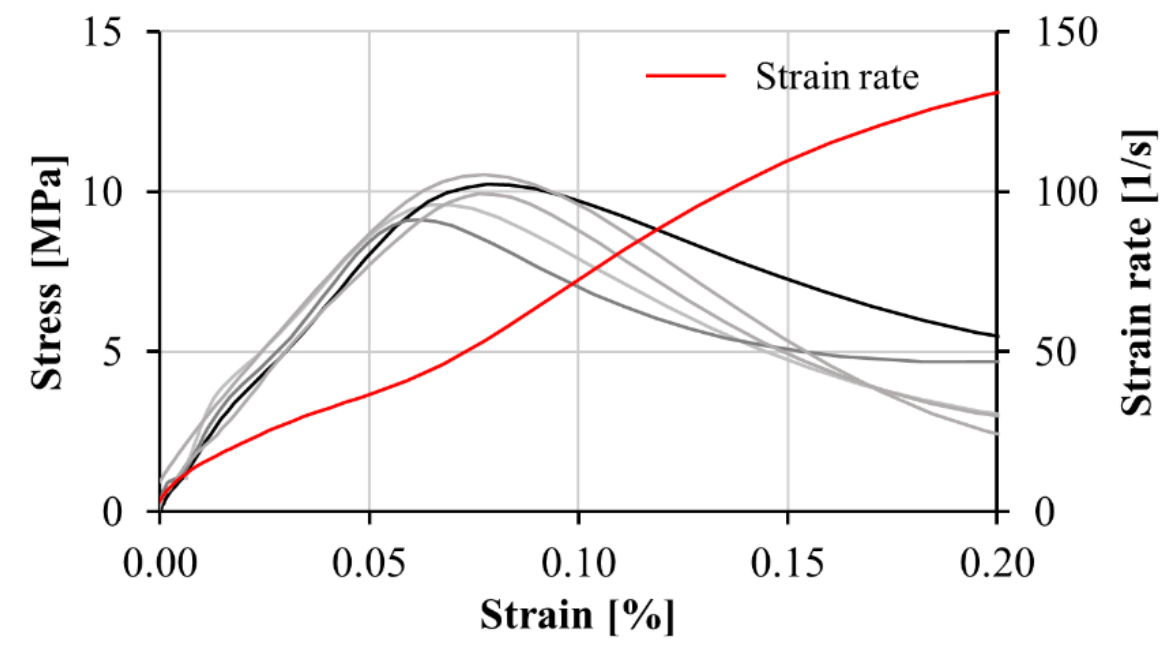

Figure 17. Stress-strain curves for SHCC specimen with a length of $20 \mathrm{~mm}$ obtained in the SHTB. 


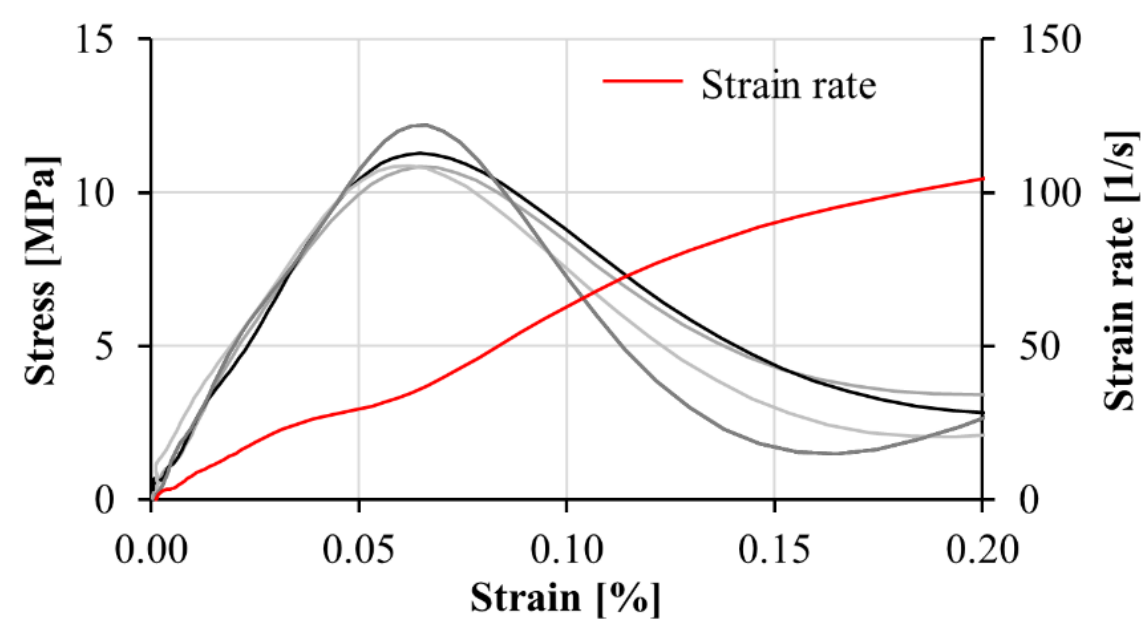

Figure 18. Stress-strain curves for annular SHCC specimen with a length of $40 \mathrm{~mm}$ obtained in the SHTB.

The results obtained from the experiments on specimens with different geometries indicate no significant change in first-crack stress in comparison to the reference experiments presented in Figure 15. It can be concluded that different mechanisms cause the apparent increase in the tensile strength of the cement-based matrix at high strain rates, mechanisms that cannot be captured in a model where the specimen is considered elastic.

As the next step, the softening and damage in the specimen were considered. Figure 19 schematically shows a specimen with a localized failure. Once the first crack starts to form, the two parts of the specimen so created accelerate in opposite directions. Initially, the whole specimen accelerates in the direction of the input bar because of the input wave. As the damage localizes in the specimen, an extra acceleration component in the direction of the input bar appears in the part connected to the input bar. Contrarily, the part connected to the transmitter bar starts to accelerate in the opposite direction toward the transmitter bar. The accelerations generated in the two seperated parts of the specimen apply extra force components to the input and the transmitter bars. These force components are shown schematically in Figure 19 with red arrows and are applied to the bars combined with the response of the material.




Figure 19. Schematic view of the inertia forces caused by the damage in the specimen.

The mechanism mentioned is observable in the results of DIC analysis. Figure 20 shows the strain field and the corresponding acceleration field on a representative SHCC specimen tested in the SHTB. The first frame on the left side shows $12.5 \mu$ s from the moment that the loading of the specimen starts. The moment of first-crack formation is shown in the last frame on the right. Frames were taken every $6.25 \mu \mathrm{s}$. It can be seen that as the wave reached the specimen in the first four frames, the whole specimen moves as one body only in the direction of the input bar. During this time, the behavior of the specimen can be considered to be elastic. Moreover, the acceleration field observed on the specimen exhibits only acceleration in the direction of the input bar. Once a crack starts to form in the specimen, the acceleration field is no longer uniform. Dark and light blue regions on the acceleration field on the upper half of the specimen indicate acceleration in the direction of the transmitter bar. The damage can be observed on the strain field as regions with light blue color, which can be considered as a forming macro-crack.

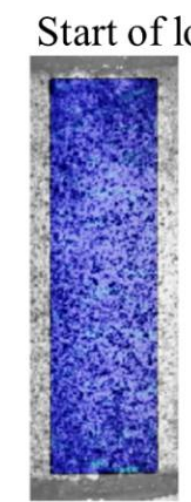

a)
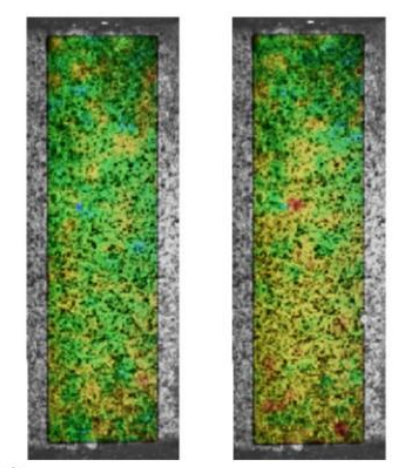


Figure 20. DIC results showing an example of a) strain field on a SHCC specimen, and b) the corresponding acceleration field. Directions of the input and the transmitter bars are shown with $+\mathrm{x}$ and $-\mathrm{x}$, respectively. 
The DIC analysis also yields the acceleration history in the direction of the input and the transmitter bar. In Figure 21, the red and the blue curves show the average acceleration in the direction of the input and the transmitter bar, $+\mathrm{x}$ and $-\mathrm{x}$, respectively. The average acceleration in each direction is calculated by taking an average over all points on the acceleration field, which show an acceleration in the respective direction. At the beginning and before the arrival of the input wave, the two curves show no acceleration. Once the wave reaches the specimen, the average acceleration in the direction of the input bar rises. However, the blue curve showing average acceleration in the direction of the transmitter bar is still at zero. Only after initiation of damage to the specimen is a rise in the blue curve observed, which shows acceleration in the direction of the transmitter bar. At the same time a sudden rise in the red curve indicates an increase of the acceleration in the direction of the input bar.

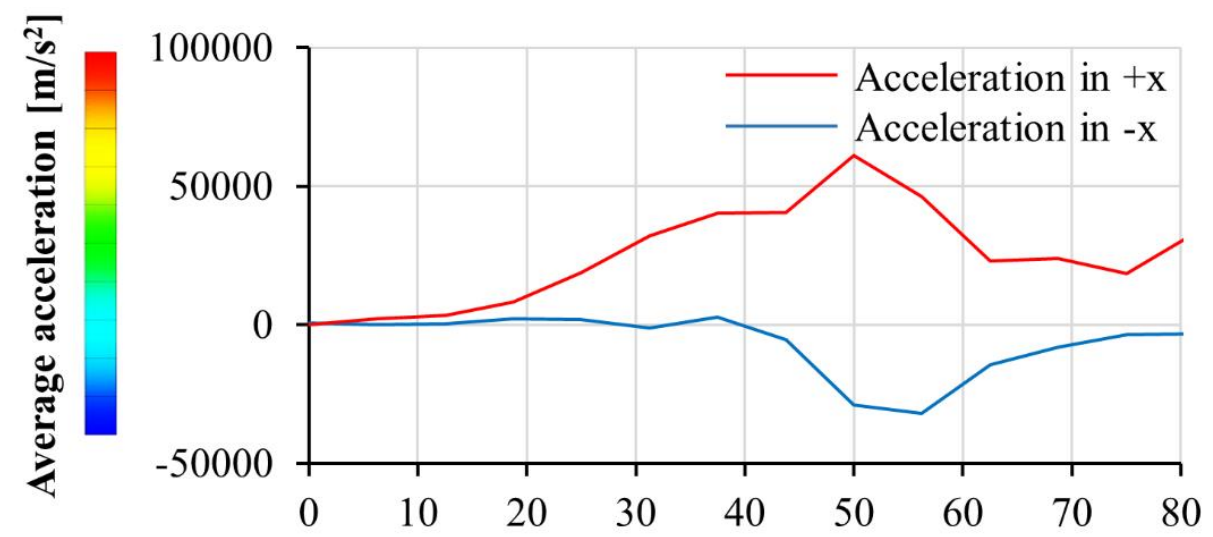

a)

Time $[\mu \mathrm{s}]$

b)

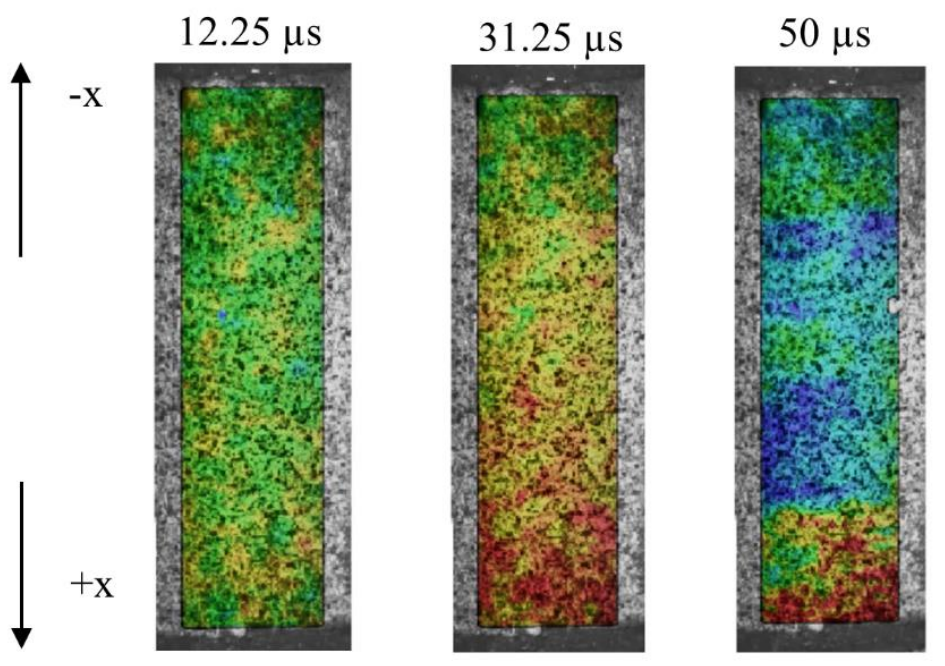

Figure 21. a) Average acceleration history in the direction of the input $(+x)$ and the transmitter bar $(-x)$, and $b)$ the corresponding DIC sequence showing acceleration distribution on the specimen. 
Taking into account the density of the material under test, the calculated average accelerations can be converted to a force component acting on the bars, as presented with red arrows in Figure 19. The accelerated mass in each direction is estimated using the number of subsets on the acceleration field, which show an acceleration in the corresponding direction. The ratio of the number of subsets showing an acceleration in the particular direction to the total number of calculated subsets can be used to estimate the mass of the specimen accelerated in that direction. It is worth noting that the average acceleration history can only be used for finding the inertia contribution to first-crack stress since at this point the specimen is only broken into two parts. By having one softening cross-section, the acceleration field is divided into two regions directly connected to the bars, showing accelerations in opposite directions. So, the resulting inertia forces are applied on the bars. However, during the multiple-cracking stage, the specimen is divided into more segments. At this stage it is not possible to calculate the exact contribution of structural inertia to the forces applied on the bars of the SHTB.



Figure 22. Contribution of inertia based on the average acceleration history, to the stress calculated on the input bar and on the transmitter bar.

The inertial force component changes over time according to the actual acceleration. Before cracking starts in the specimen, the contribution of inertia is only notable on the input bar; see the red curve in Figure 22. After $37.5 \mu$ s, a crack develops, and a considerable contribution of inertia on the transmitter bar is observed; see the blue curve in Figure 22. The contribution of the inertia forces to the average stress calculated on the bars based on the recorded waves, shown by the black curve in Figure 22, reaches $17 \%$ at $50 \mu$ s at the peak stress. For all the specimens tested in SHTB, the contributions of inertia were calculated using DIC results following the same method. Table 6 provides the average contribution of inertia to first-crack stress in the SHCC specimens and to the tensile strength in the plain matrix specimens. The average contribution of inertia to the tensile strength of the matrix obtained in non-reinforced 
matrix specimens was higher than that observed as the first-crack stress of SHCC specimens. The bridging action of the fibers and their influence in reducing the accelerations created due to localized failure in the specimen is the reason behind the lower contribution of inertia to firstcrack stress of the SHCC.

Table 6. Average contribution of inertia to the tensile strength of the cement-based matrix and first-crack stress of SHCC obtained in SHTB. Standard deviation is given in the parentheses.

\begin{tabular}{|l|l|}
\hline \multicolumn{2}{|l|}{ Non-reinforced matrix } \\
\hline Tensile strength [MPa] & $9.8(1.8)$ \\
\hline Contribution of structural inertia [\%] & $28(3.8)$ \\
\hline SHCC & $10.3(0.9)$ \\
\hline First-crack stress [MPa] & $12(2.9)$ \\
\hline Contribution of structural inertia [\%]
\end{tabular}

\subsection{Micromechanical investigations}

\subsubsection{Results of quasi-static fiber pullout tests}

The force-displacement relations of quasi-static, single fiber pullout experiments are presented in Figure 23. The quasi-static tests were continued until the point of complete pullout of the fibers at $4 \mathrm{~mm}$ displacement. However, the displacement applied by the input wave in the miniHopkinson bar was limited to $0.6 \mathrm{~mm}$. For better comparison, all force-displacement curves are only shown up to $0.6 \mathrm{~mm}$ displacement. The displacement of $0.6 \mathrm{~mm}$ was sufficient for capturing the initial debonding phase as well as the frictional pullout force.

The quasi-static force-displacement curves in Figure $\mathbf{2 3}$ show a linear increase of the force at the beginning. This increase of force against pullout displacement occurs during debonding of the fiber from the surrounding matrix. It can be assumed that peak pullout force corresponds to the moment of complete debonding, from which on the fiber starts to move in the channel. Once the fiber is completely debonded from the matrix, the pullout phase starts, where the friction force between the fiber's surface and the matrix causes the pullout resistance [7]. Since the contact area between fiber and matrix decreases gradually as displacement increases, the friction force decreases as well. Note that the hydrophobic nature of the HDPE fibers used in this study prevents them from forming a strong bond with cement-based matrix. 




Figure 23. Force-displacement relations obtained in the quasi-static pullout of single HDPE fiber from the cement matrix.

\subsubsection{Results of high-speed fiber pullout tests}

The force-displacement curves obtained in the high-speed pullout experiments showed an average peak pullout force of $0.38 \mathrm{~N}$, which yields a DIF of 5.7; see Figure 24. The average post-peak frictional pullout force obtained in the high-speed experiments was also considerably higher than those obtained in the quasi-static experiments, providing a DIF of 2.7. The results of the quasi-static and high-speed experiments are summarized in Table 7. The post-peak frictional pullout force in both loading regimes was calculated by averaging the force values obtained for displacements between 0.2 and $0.6 \mathrm{~mm}$.

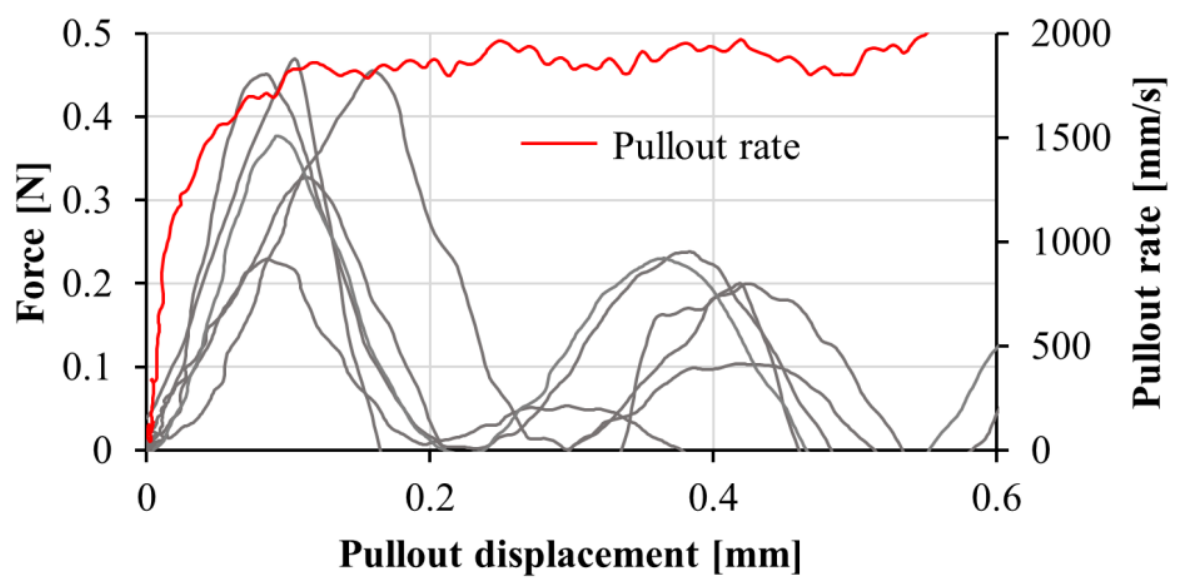

Figure 24. Force-displacement relations obtained in the high-speed pullout of single HDPE fiber from the cement-based matrix performed in the mini split Hopkinson tension bar.

The fiber-pullout mechanism in high-speed pullout experiments can be assumed to be similar to that in quasi-static tests. However, the rate-dependent behavior of the fiber itself may influence the pullout behavior. It was shown that Young's modulus and tensile strength of the 
fiber increase with increasing strain rate [7]. The viscous nature of the polymeric fibers can be the reason behind their rate-dependent behavior. The higher stiffness and strength of the fiber at higher loading rates increase its resistance against damage and deformation during the pullout process. The result is a higher pullout force at higher displacement rates.

Table 7. Results of quasi-static and high-speed pullout of single PE fiber from M1 matrix; standard deviations are given in parantheses.

\begin{tabular}{|l|l|}
\hline \multicolumn{2}{|l|}{ Quasi-static } \\
\hline Peak pullout force [N] & $0.07(0.05)$ \\
\hline Frictional pullout force [N] & $0.03(0.02)$ \\
\hline High-speed & $0.4(0.08)$ \\
\hline Peak pullout force [N] & 5.7 \\
\hline DIF of peak pullout force & $0.08(0.04)$ \\
\hline Frictional pullout force [N] & 2.7 \\
\hline DIF of frictional pullout force &
\end{tabular}

It must be noted that the very high DIF obtained here for the bond strength was not observed for the tensile strength of the composite tested under impact loading. Although tensile strength is directly related to fiber-matrix bond strength and the bridging capacity of the fibers, the DIF of the composite tensile strength was only 1.8; see Table 5. The reason is the fact that cracks in the composite open very fast at the beginning, but thereafter the opening speed reduces gradually as other cracks are formed in the multiple cracking phase. This phenomenon is illustrated in Figure 25. A number of cracks in a SHCC specimen tested on the SHTB have been selected. It can be seen that their opening speed reaches a very high value at the beginning. The crack-opening speed at this stage is comparable to pullout speed generated on the miniHopkinson bar. However, upon the formation of new cracks, the opening speed of the "old" ones drop rapidly. As a consequence, the pullout force of the fibers in the composite is not as high as that observed in high-speed pullout tests, where the pullout speed remains high for the whole duration of the test. It should also be noted that the inertia effects in dynamic fiber pullout experiments and the recorded force can also have an influence on the DIF value of the pullout force. However, an investigation of this issue is not performed in the current work. 

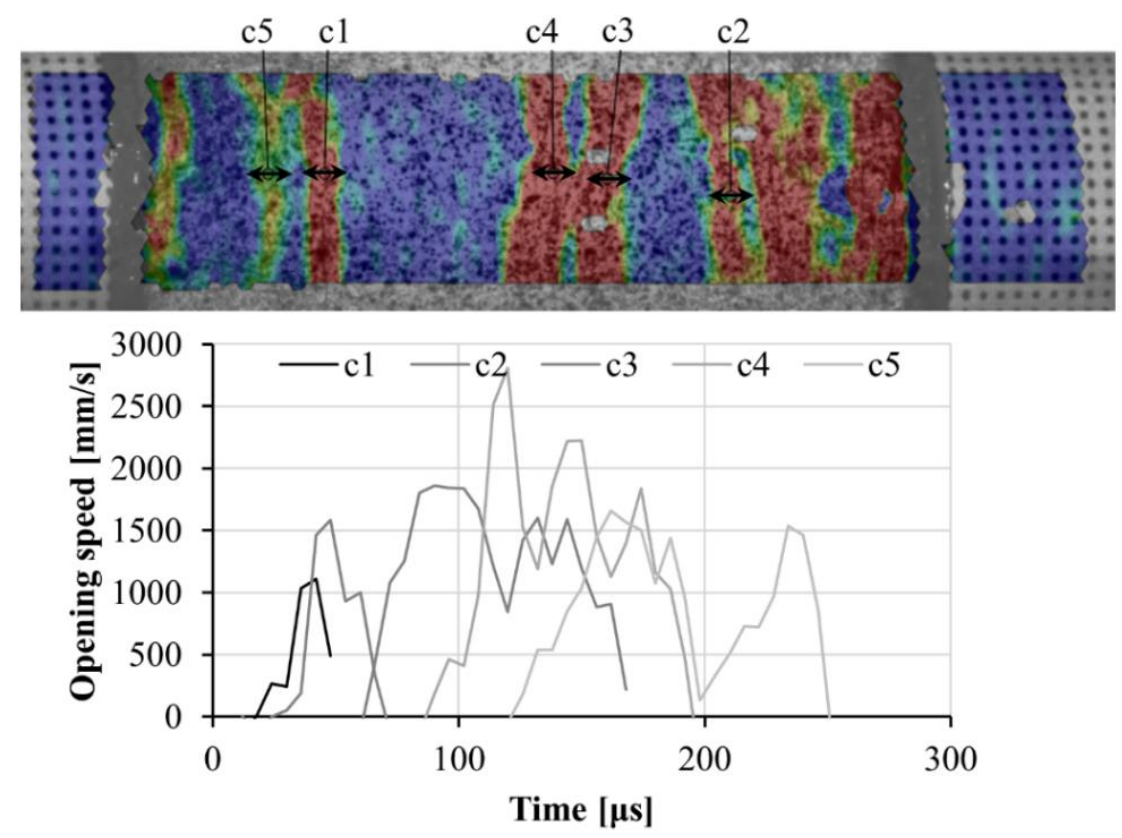

Figure 25. Crack-opening speed of five cracks formed in a SHCC specimen tested in the SHTB; the numbering order of the cracks represents their generation order in the specimen.

\section{Conclusions}

The behavior of SHCC under impact loading was studied on the composite-scale as well as the microscale. A split Hopkinson tension bar was used to characterize SHCC under impact tensile load. Moreover, the rate-dependent bond between fiber and matrix was studied using a miniaturized split Hopkinson tension bar.

First-crack stress of SHCC specimens and the tensile strength of the matrix specimens under impact loading were found to be considerably higher than those obtained in quasi-static tests. With the help of high-speed imaging, digital image correlation (DIC), and experiments on specimens with different geometries, an investigation into the influence of structural inertia was performed. Analysis of the results indicates that the conventional analysis of the structural inertial effects, assuming elastic deformation in the specimen up to the formation of the first crack, results in underestimating the contribution of structural inertia. It was shown that the damage and softening of the material could cause considerable inertial forces on both bars of SHTB. The inertial forces were generated due to the acceleration caused by damage and are incorrectly included in the material response derived. Thus, a novel method based on the DIC results was used to differentiate the influence of structural inertia from the rate-sensitive material response.

Under impact loading, the SHCC tested showed improved multiple cracking, strain capacity and energy absorption capacity. The results obtained from dynamic fiber-pullout tests were 
used to explain the improved behavior of the composite under impact loading. The significantly improved fiber-matrix bond explains the enhanced crack-bridging action at high strain rates. Furthermore, DIC results show mechanisms that enable the formation of multiple cracks at stress levels lower than that of the first crack. Firstly, the distributed damage, which happens in the cement-based matrix before the formation of the first crack, can explain the lower cracking stress in the multiple cracking stage. Secondly, the dramatic reduction of strain rate in the uncracked regions, which occurs during the multiple cracking stage, limits the influence of rate sensitivity on the cracking stress of the matrix. The experimental results presented not only provide a thorough study of the behavior of SHCC under dynamic tensile loading but also provide a basis to develop methodologies for optimization and tailoring SHCC mixtures for such loads.

\section{Acknowledgement}

The authors gratefully acknowledge the financial support of the German Research Foundation (DFG) for funding the Research Training Group GRK 2250 "Mineral-bonded composites for enhanced structural impact safety".

\section{References}

[1] I. Curosu, M. Liebscher, V. Mechtcherine, C. Bellmann, and S. Michel, "Tensile behavior of high-strength strain-hardening cement-based composites (HS-SHCC) made with high-performance polyethylene, aramid and PBO fibers," Cem. Concr. Res., vol. 98, pp. 71-81, 2017.

[2] V. C. Li, "On engineered cementitious composites (ECC) a review of the material and its applications," J. Adv. Concr. Technol., vol. 1, no. 3, pp. 215-230, 2003.

[3] V. Mechtcherine, "Novel cement-based composites for the strengthening and repair of concrete structures," Constr. Build. Mater., vol. 41, pp. 365-373, 2013.

[4] I. Curosu, V. Mechtcherine, M. Hering, and M. Curbach, "Mineral-bonded composites for enhanced structural impact safety - Overview of the format, goals and achievements of the research group GRK 2250," in FraMCoS-X: 10th International Conference on Fracture Mechanics of Concrete and Concrete Structures, 2019.

[5] I. Curosu, V. Mechtcherine, D. Forni, and E. Cadoni, "Performance of various strainhardening cement-based composites (SHCC) subject to uniaxial impact tensile loading," Cem. Concr. Res., vol. 102, pp. 16-28, 2017.

[6] A. A. Heravi, I. Curosu, and V. Mechtcherine, “A gravity-driven split Hopkinson tension 
bar for investigating quasi-ductile and strain-hardening cement-based composites under tensile impact loading," Cem. Concr. Compos., vol. 105, p. 103430, 2019.

[7] I. Curosu, V. Mechtcherine, and O. Millon, "Effect of fiber properties and matrix composition on the tensile behavior of strain-hardening cement-based composites (SHCCs) subject to impact loading," Cem. Concr. Res., vol. 82, pp. 23-35, 2016.

[8] E. H. Yang and V. C. Li, "Tailoring engineered cementitious composites for impact resistance," Cem. Concr. Res., vol. 42, no. 8, pp. 1066-1071, 2012.

[9] A. Caverzan, E. Cadoni, and M. Di Prisco, "Tensile behaviour of high performance fibrereinforced cementitious composites at high strain rates," Int. J. Impact Eng., vol. 45, pp. $28-38,2012$.

[10] P. Rossi and F. Toutlemonde, "Effect of loading rate on the tensile behaviour of concrete : Description of the physical mechanisms," Mater. Struct. Constr., vol. 29, no. 186, pp. 116-118, 1996.

[11] M. Zhang, H. J. Wu, Q. M. Li, and F. L. Huang, "Further investigation on the dynamic compressive strength enhancement of concrete-like materials based on split Hopkinson pressure bar tests . Part I : Experiments," Int. J. Impact Eng., vol. 36, no. 12, pp. 1327 1334, 2009.

[12] U. Häussler-combe and E. Panteki, "Modeling of concrete spallation with damaged viscoelasticity and retarded damage,” Int. J. Solids Struct., vol. 90, pp. 153-166, 2016.

[13] L. F. Pereira, J. Weerheijm, and L. J. Sluys, "A new effective rate dependent damage model for dynamic tensile failure of concrete," Eng. Fract. Mech., vol. 176, pp. 281299, 2017.

[14] Y. B. Lu and Q. M. Li, “About the dynamic uniaxial tensile strength of concrete-like materials," Int. J. Impact Eng., vol. 38, no. 4, pp. 171-180, 2011.

[15] H. W. Reinhardt and J. Weerheijm, "Tensile fracture of concrete at high loading rates taking account of inertia and crack velocity effects," Int. J. Fract., vol. 51, no. 1, pp. 3142, 1991.

[16] J. Ožbolt, A. Sharma, and H.-W. Reinhardt, "Dynamic fracture of concrete - compact tension specimen," Int. J. Solids Struct., vol. 48, no. 10, pp. 1534-1543, 2011.

[17] J. Ožbolt, J. Bošnjak, and E. Sola, "Dynamic fracture of concrete compact tension specimen: Experimental and numerical study," Int. J. Solids Struct., vol. 50, no. 25, pp. 
4270-4278, 2013.

[18] J. Ožbolt, A. Sharma, B. İrhan, and E. Sola, "Tensile behavior of concrete under high loading rates," Int. J. Impact Eng., vol. 69, pp. 55-68, 2014.

[19] A. C. C. Trindade, A. A. Heravi, I. Curosu, M. Liebscher, D. Andrade, and V. Mechtcherine, "Tensile behavior of strain-hardening geopolymer composites ( SHGC) under impact loading," Cem. Concr. Compos., vol. 113, p. 103703, 2020.

[20] A. A. Heravi, A. Fuchs, T. Gong, I. Curosu, M. Kaliske, and V. Mechtcherine, "Mechanical characterization of textile reinforced cementitious composites under impact tensile loading using the split Hopkinson tension bar," Cem. Concr. Compos., vol. 114, p. 103769, 2020.

[21] H. Liao and W. W. Chen, "Specimen-Bar Impedance Mismatch Effects on Equilibrium and Rate Constancy for Kolsky Bar Experiments," Exp. Mech., vol. 58, no. 9, pp. 14391449, 2018.

[22] B. Song and W. Chen, "Dynamic stress equilibration in split Hopkinson pressure bar tests on soft materials," Exp. Mech., vol. 44, no. 3, pp. 300-312, 2004.

[23] A. Chatterjee and A. L. Ruina, "Two Interpretations of Rigidity in Rigid-Body Collisions," J. Appl. Mech., vol. 65, no. 4, p. 894, 1998.

[24] F. E. Hauser, "Techniques for measuring stress-strain relations at high strain rates," Exp. Mech., vol. 6, no. 8, pp. 395-402, 1966.

[25] G. Haugou, N. Leconte, and H. Morvan, "Design of a pre-stretched tension Hopkinson bar device : Configuration, tail corrections, and numerical validation," Int. J. Impact Eng., vol. 97, pp. 89-101, 2016.

[26] E. Jacquelin and P. Hamelin, "Block-bar device for energy absorption analysis," Mech. Syst. Signal Process., vol. 15, no. 3, pp. 603-617, 2001.

[27] B. Lundberg and A. Henchoz, "Analysis of elastic waves from two-point strain measurement," Exp. Mech., vol. 17, no. 6, pp. 213-218, 1977.

[28] M. N. Bussac, P. Collet, G. Gary, and R. Othman, "An optimisation method for separating and rebuilding one-dimensional dispersive waves from multi-point measurements. Application to elastic or viscoelastic bars," J. Mech. Phys. Solids, vol. 50, no. 2, pp. 321-349, 2002.

[29] G. Gary, "Testing With Bars From Dynamic to Quasi-static," in Constitutive Relations 
under Impact Loadings, Springer, Vienna, 2014, pp. 1-58.

[30] L. He, G. W. Ma, B. Karp, and Q. M. Li, "Investigation of dynamic Saint-Venant's principle in a cylindrical waveguide - Analytical results," Int. J. Impact Eng., vol. 73, pp. 135-144, 2014.

[31] D. Mohr, G. Gary, and B. Lundberg, "Evaluation of stress-strain curve estimates in dynamic experiments," Int. J. Impact Eng., vol. 37, no. 2, pp. 161-169, 2010.

[32] M. Ranjbarian and V. Mechtcherine, "A novel test setup for the characterization of bridging behaviour of single microfibres embedded in a mineral-based matrix," Cem. Concr. Compos., vol. 92, pp. 92-101, 2018.

[33] J. Lim, W. W. Chen, and J. Q. Zheng, "Dynamic small strain measurements of Kevlar 129 single fi bers with a miniaturized tension Kolsky bar," Polym. Test., vol. 29, no. 6, pp. 701-705, 2010.

[34] B. Sanborn and T. Weerasooriya, "Quantifying damage at multiple loading rates to Kevlar KM2 fibers due to weaving, finishing, and pre-twist," Int. J. Impact Eng., vol. 71, pp. 50-59, 2014.

[35] M. Larcher, "Development of discrete cracks in concrete loaded by shock waves," Int. J. Impact Eng., vol. 36, no. 5, pp. 700-710, 2009.

[36] P. Rossi, "Influence of cracking in the presence of free water on the mechanical behaviour of concrete," Mag. Concr. Res., vol. 43, no. 154, pp. 53-57, 1991.

[37] A. Brara and J. R. Klepaczko, "Experimental characterization of concrete in dynamic tension," Mech. Mater., vol. 38, no. 3, pp. 253-267, 2006.

[38] P. Forquin, "An optical correlation technique for characterizing the crack velocity in concrete,”Eur. Phys. J. Spec. Top., vol. 206, no. 1, pp. 89-95, 2012.

[39] E. Binder, R. Reihsner, Y. Yuan, H. A. Mang, and B. L. A. Pichler, "High-dynamic compressive and tensile strength of specimens made of cementitious materials," Cem. Concr. Res., vol. 129, p. 105890, 2020.

[40] R. Othman, "Inertial and Frictional Effects in Dynamic Compression Testing," in The Kolsky- Hopkinson Bar Machine: selected topics, Springer, 2018, pp. 205-249.

[41] D. A. Gorham, "Specimen inertia in high strain-rate compression," J. Phys. D. Appl. Phys., vol. 22, no. 12, pp. 1888-1893, 1989.

[42] M. J. Forrestal, T. W. Wright, and W. Chen, "The effect of radial inertia on brittle 
samples during the split Hopkinson pressure bar test," Int. J. Impact Eng., vol. 34, no. 3, pp. 405-411, 2007.

[43] M. Hockly and C. R. Siviour, "Specimen Inertia in high strain rate tensile testing," EPJ Web Conf., vol. 94, 2015. 